Network Working Group

Request for Comments: 3830

Category: Standards Track
J. Arkko

E. Carrara

F. Lindholm

M. Naslund

K. Norrman

Ericsson Research

August 2004

\title{
MIKEY: Multimedia Internet KEYing
}

Status of this Memo

This document specifies an Internet standards track protocol for the Internet community, and requests discussion and suggestions for improvements. Please refer to the current edition of the "Internet Official Protocol Standards" (STD 1) for the standardization state and status of this protocol. Distribution of this memo is unlimited.

Copyright Notice

Copyright (C) The Internet Society (2004).

Abstract

This document describes a key management scheme that can be used for real-time applications (both for peer-to-peer communication and group communication). In particular, its use to support the Secure Realtime Transport Protocol is described in detail.

Security protocols for real-time multimedia applications have started to appear. This has brought forward the need for a key management solution to support these protocols. 
Table of Contents

1. Introduction . . . . . . . . . . . . . . . . . . . . . . . . . 3

1.1. Existing Solutions . . . . . . . . . . . . . . . . . . . 4 4

1.2. Notational Conventions . . . . . . . . . . . . . . . . 4 4

1.3. Definitions. . . . . . . . . . . . . . . . . . . . 4 4

1.4. Abbreviations. . . . . . . . . . . . . . . . . 6

1.5. Outline. . . . . . . . . . . . . . . . . . . 6

2. Basic Overview . . . . . . . . . . . . . . . . . . 7

2.1. Scenarios. . . . . . . . . . . . . . . . . . . . . . . .7

2.2. Design Goals. . . . . . . . . . . . . . . . . . . 8

2.3. System Overview. . . . . . . . . . . . . . . . . . 8

2.4. Relation to GKMARCH. . . . . . . . . . . . . . . 10

3. Basic Key Transport and Exchange Methods . . . . . . . . . . 10

3.1. Pre-shared Key . . . . . . . . . . . . . . . . 12

3.2. Public-Key Encryption. . . . . . . . . . . . . . . 13

3.3. Diffie-Hellman Key Exchange. • • • • • • • • • • • • • 14

4. Selected Key Management Functions. . . . . . . . . . . . . . 15

4.1. Key Calculation. . . . . . . . . . . . . . . . . 16

4.1.1. Assumptions. . . . . . . . . . . . . . 16

4.1.2. Default PRF Description. . . . . . . . . . . 17

4.1.3. Generating keys from TGK . . . . . . . . . . 18

4.1.4. Generating keys for MIKEY Messages from

an Envelope/Pre-Shared Key . . . . . . . . 19

4.2 Pre-defined Transforms and Timestamp Formats . . . . . . 19

4.2.1. Hash Functions . . . . . . . . . . . . . . . . . 19

4.2.2. Pseudo-Random Number Generator and PRF . . . . 20

4.2.3. Key Data Transport Encryption. . . . . . . . . . 20

4.2.4. MAC and Verification Message Function. . . . . . 21

4.2.5. Envelope Key Encryption. . . . . . . . . . . . . 21

4.2.6. Digital Signatures . . . . . . . . . . . . . 21

4.2.7. Diffie-Hellman Groups. . . . . . . . . . . . 21

4.2.8. Timestamps . . . . . . . . . . . . . . . . 21

4.2.9. Adding New Parameters to MIKEY . . . . . . . . 22

4.3. Certificates, Policies and Authorization . . . . . . . 22

4.3.1. Certificate Handling . . . . . . . . . . . 22

4.3.2. Authorization. . . . . . . . . . . . 23

4.3.3. Data Policies. . . . . . . . . . . . . . . . 24

4.4. Retrieving the Data SA . . . . . . . . . . . . . . . 24

4.5. TGK Re-Keying and CSB Updating . . . . . . . . . . . . 25

5. Behavior and Message Handling. . . . . . . . . . . . . . 26

5.1. General. . . . . . . . . . . . . . . . . . . 26

5.1.1. Capability Discovery . • . • • . • . • . . 26

5.1.2. Error Handling . . . . . . . . . . . . 27

5.2. Creating a Message . . . . . . . . . . . . . . 28

5.3. Parsing a Message. . . . . . . . . . . . . . . . . . 29

5.4. Replay Handling and Timestamp Usage. . . . . . . . . . 30

6. Payload Encoding . . . . . . . . . . . . . . . . 32 
6.1. Common Header Payload (HDR) . . . . . . . . . . . . . 32

6.1.1. SRTP ID. . . . . . . . . . . . . . . . 35

6.2. Key Data Transport Payload (KEMAC) . . . . . . . . . 36

6.3. Envelope Data Payload (PKE) . . . . . . . . . . . . 37

6.4. DH Data Payload (DH) . . . . . . . . . . . . . . 38

6.5. Signature Payload (SIGN) . . . . . . . . . . . . . . . 39

6.6. Timestamp Payload (T). . . . . . . . . . . . . . 39

6.7. ID Payload (ID) / Certificate Payload (CERT) . • • . . 40

6.8. Cert Hash Payload (CHASH). . . . . . . . . . . . . . . 41

6.9. Ver msg payload (V). . . . . . . . . . . . . . . . . 42

6.10. Security Policy Payload (SP) . . . . . . . . . . . . . 42 6.10.1. SRTP Policy. . . . . . . . . . . . . . . 44

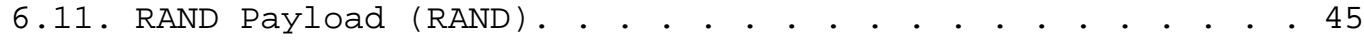

6.12. Error Payload (ERR). . . . . . . . . . . . . . . . 46

6.13. Key Data Sub-Payload . . . . . . . . . . . . . . 46

6.14. Key Validity Data. . . . . . . . . . . . . . . . . 48

6.15. General Extension Payload. . . . • . • • • • • • . . 50

7. Transport Protocols. . . . . . . . . . . . . . . . 50

8. Groups . . . . . . . . . . . . . . . . . . . . . . . 50

8.1. Simple One-to-Many . . . . . . . . . . . . . . . . . . 51

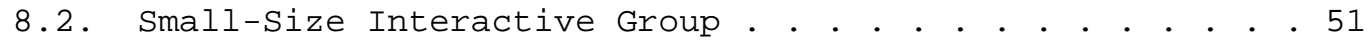

9. Security Considerations. . . . . . . . . . . . . . . . . 52

9.1. General. . . . . . . . . . . . . . . . . . . . 52

9.2. Key Lifetime. . . . . . . . . . . . . . . . . . . 54

9.3. Timestamps . . . . . . . . . . . . . . . . . . 55

9.4. Identity Protection. . . . . . . . . . . . . . . 55

9.5. Denial of Service. . . . . . . . . . . . . . . 56

9.6. Session Establishment. . . . . . . . . . . . . . 56

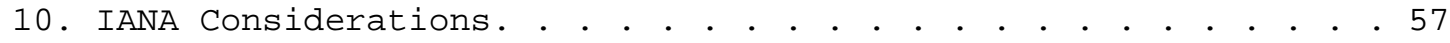

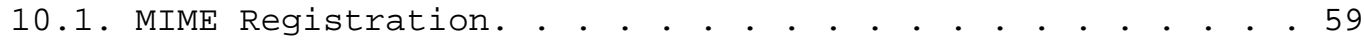

11. Acknowledgments. . . . . . . . . . . . . . . . . . . 59

12. References . . . . . . . . . . . . . . . . . . . 60

12.1. Normative References . . . . . . . . . . . . . . 60

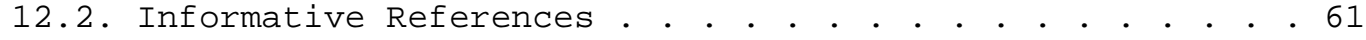

Appendix A. - MIKEY - SRTP Relation. . . . . . . . . . . . . . 63

Author's Addresses . . . . . . . . . . . . . . . . . . . 65

Full Copyright Statement . . . . . . . . . . . . . . . 66

\section{Introduction}

There has recently been work to define a security protocol for the protection of real-time applications running over RTP, [SRTP] . However, a security protocol needs a key management solution to exchange keys and related security parameters. There are some fundamental properties that such a key management scheme has to fulfill to serve streaming and real-time applications (such as unicast and multicast), particularly in heterogeneous (mix of wired and wireless) networks. 
This document describes a key management solution that addresses multimedia scenarios (e.g., SIP [SIP] calls and RTSP [RTSP] sessions). The focus is on how to set up key management for secure multimedia sessions such that requirements in a heterogeneous environment are fulfilled.

\subsection{Existing Solutions}

There is work done in the IETF to develop key management schemes. For example, IKE [IKE] is a widely accepted unicast scheme for IPsec, and the MSEC WG is developing other schemes to address group communication [GDOI, GSAKMP]. However, for reasons discussed below, there is a need for a scheme with lower latency, suitable for demanding cases such as real-time data over heterogeneous networks and small interactive groups.

An option in some cases might be to use [SDP], as SDP defines one field to transport keys, the "k=" field. However, this field cannot be used for more general key management purposes, as it cannot be extended from the current definition.

\subsection{Notational Conventions}

The key words "MUST", "MUST NOT", "REQUIRED", "SHALL", "SHALL NOT", "SHOULD", "SHOULD NOT", "RECOMMENDED", "MAY", and "OPTIONAL" in this document are to be interpreted as described in BCP 14, RFC 2119 [RFC2119].

\subsection{Definitions}

(Data) Security Protocol: the security protocol used to protect the actual data traffic. Examples of security protocols are IPsec and SRTP.

Data Security Association (Data SA): information for the security protocol, including a TEK and a set of parameters/policies.

Crypto Session (CS): uni- or bi-directional data stream(s), protected by a single instance of a security protocol. For example, when SRTP is used, the crypto session will often contain two streams, an RTP stream and the corresponding RTCP, which are both protected by a single SRTP Cryptographic Context, i.e., they share key data and the bulk of security parameters in the SRTP Cryptographic context (default behavior in [SRTP]). In the case of IPsec, a Crypto Session would represent an instantiation of an IPsec SA. A Crypto Session can be viewed as a Data SA (as defined in [GKMARCH]) and could therefore be mapped to other security protocols if necessary. 


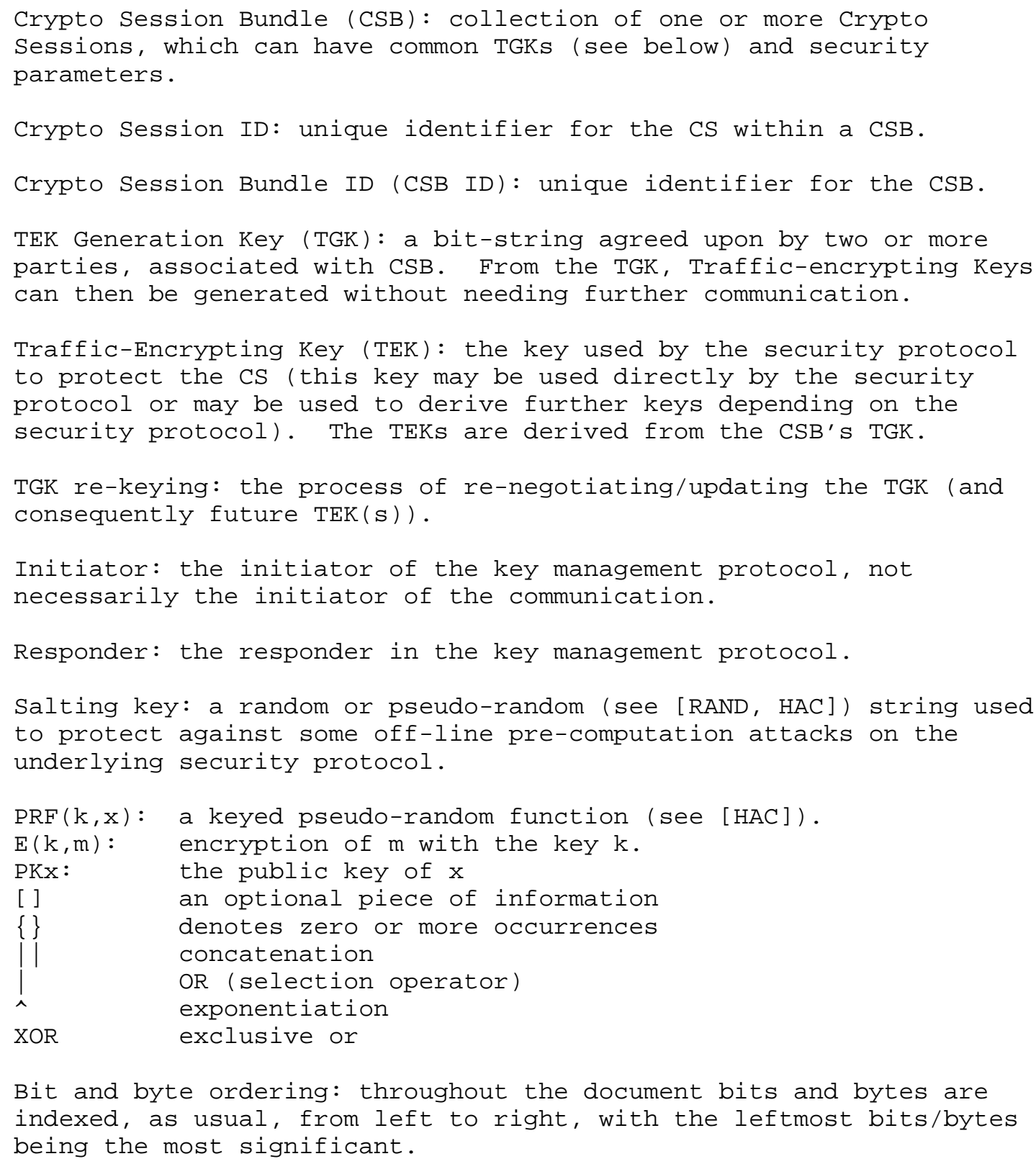




\subsection{Abbreviations}

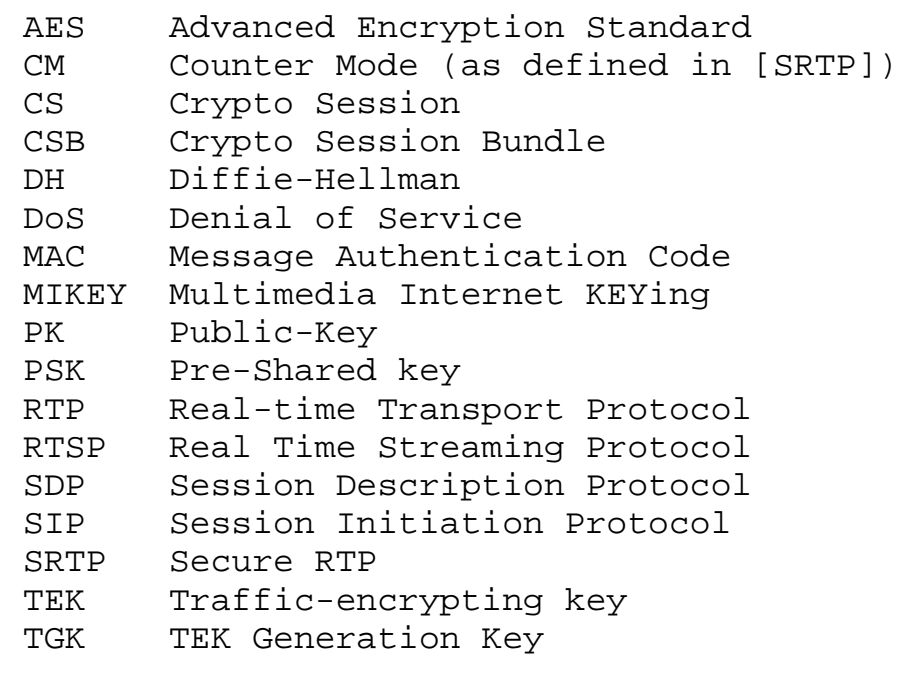

1.5. Outline

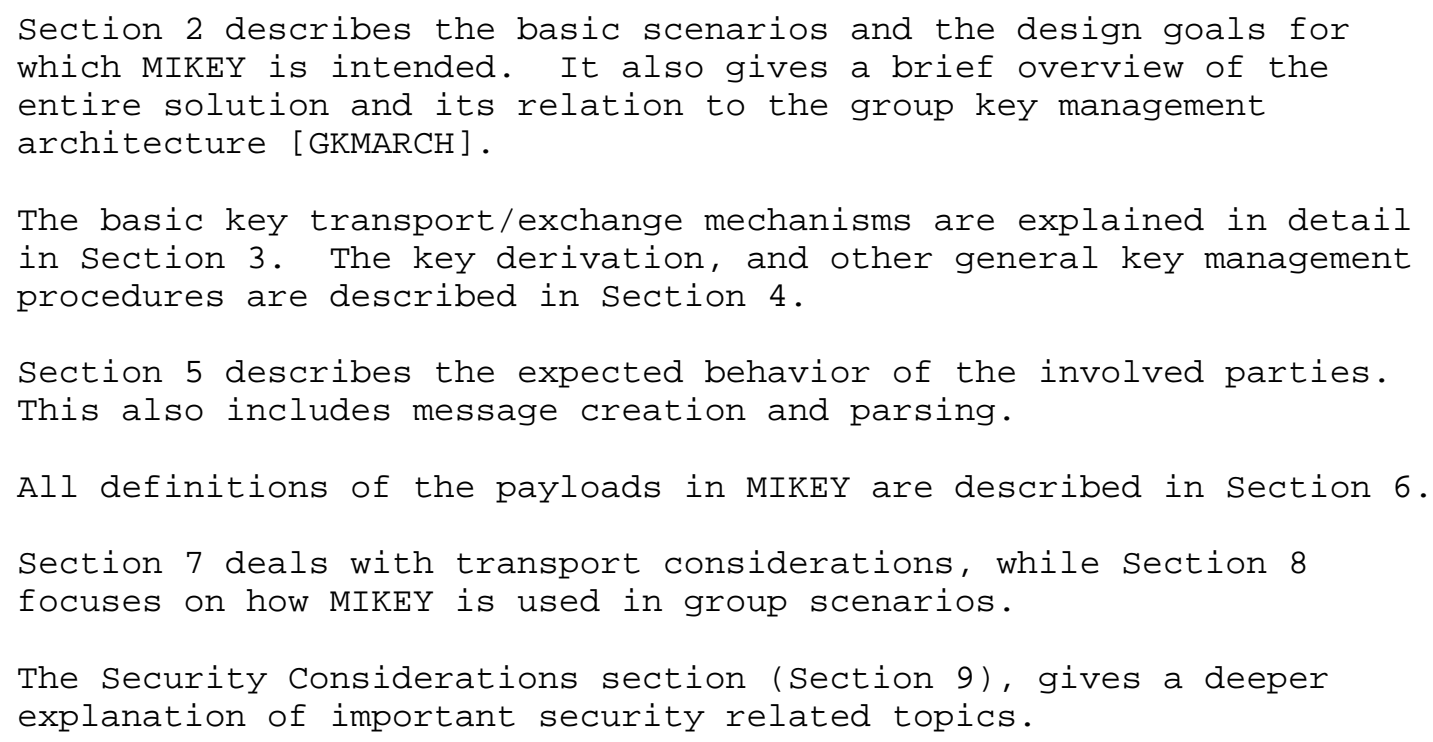




\section{Basic Overview}

\subsection{Scenarios}

MIKEY is mainly intended to be used for peer-to-peer, simple one-tomany, and small-size (interactive) groups. One of the main multimedia scenarios considered when designing MIKEY has been the conversational multimedia scenario, where users may interact and communicate in real-time. In these scenarios it can be expected that peers set up multimedia sessions between each other, where a multimedia session may consist of one or more secured multimedia streams (e.g., SRTP streams).
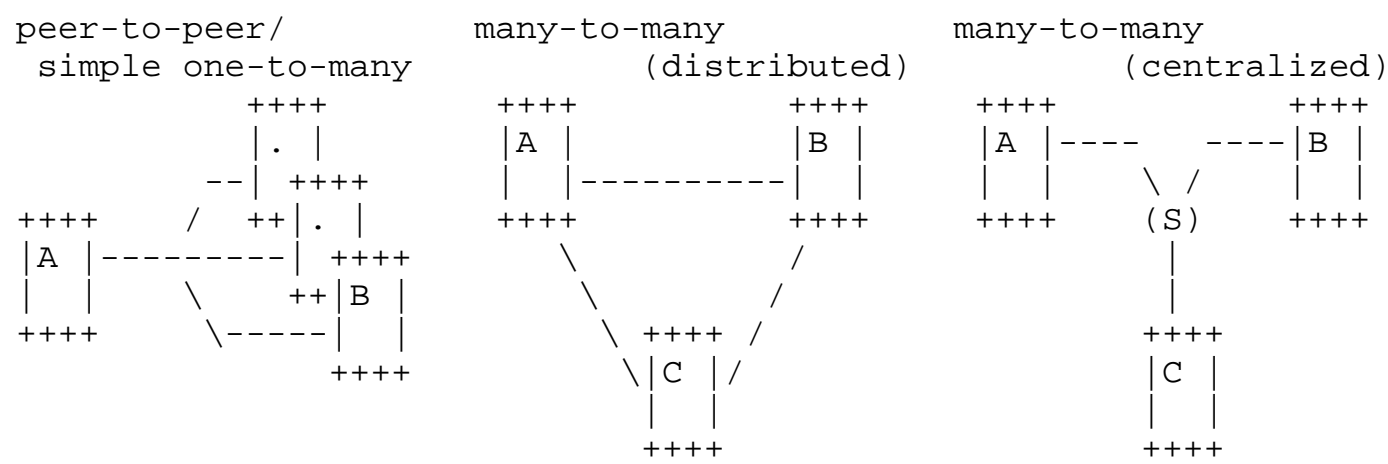

Figure 2.1: Examples of the four scenarios: peer-to-peer, simple one-to-many, many-to-many without a centralized server (also denoted as small interactive group), and many-to-many with a centralized server.

We identify in the following some typical scenarios which involve the multimedia applications we are dealing with (see also Figure 2.1).

a) peer-to-peer (unicast), e.g., a SIP-based [SIP] call between two parties, where it may be desirable that the security is either set up by mutual agreement or that each party sets up the security for its own outgoing streams.

b) simple one-to-many (multicast), e.g., real-time presentations, where the sender is in charge of setting up the security.

c) many-to-many, without a centralized control unit, e.g., for small-size interactive groups where each party may set up the security for its own outgoing media. Two basic models may be used here. In the first model, the Initiator of the group acts as the 
group server (and is the only one authorized to include new members). In the second model, authorization information to include new members can be delegated to other participants.

d) many-to-many, with a centralized control unit, e.g., for larger groups with some kind of Group Controller that sets up the security.

The key management solutions may be different in the above scenarios. When designing MIKEY, the main focus has been on case $a, b$, and $c$. For scenario $c$, only the first model is covered by this document.

\subsection{Design Goals}

The key management protocol is designed to have the following characteristics:

* End-to-end security. Only the participants involved in the communication have access to the generated key(s).

* Simplicity.

* Efficiency. Designed to have:

- low bandwidth consumption,

- low computational workload,

- small code size, and

- minimal number of roundtrips.

* Tunneling. Possibility to "tunnel"/integrate MIKEY in session establishment protocols (e.g., SDP and RTSP).

* Independence from any specific security functionality of the underlying transport.

\subsection{System Overview}

One objective of MIKEY is to produce a Data SA for the security protocol, including a traffic-encrypting key (TEK), which is derived from a TEK Generation Key (TGK), and used as input for the security protocol.

MIKEY supports the possibility of establishing keys and parameters for more than one security protocol (or for several instances of the same security protocol) at the same time. The concept of crypto Session Bundle (CSB) is used to denote a collection of one or more Crypto sessions that can have common TGK and security parameters, but which obtain distinct TEKs from MIKEY. 
The procedure of setting up a CSB and creating a TEK (and Data SA), is done in accordance with Figure 2.2:

1. A set of security parameters and TGK(s) are agreed upon for the Crypto Session Bundle (this is done by one of the three alternative key transport/exchange mechanisms, see section 3).

2. The TGK(s) is used to derive (in a cryptographically secure way) a TEK for each Crypto Session.

3. The TEK, together with the security protocol parameters, represent the Data SA, which is used as the input to the security protocol.

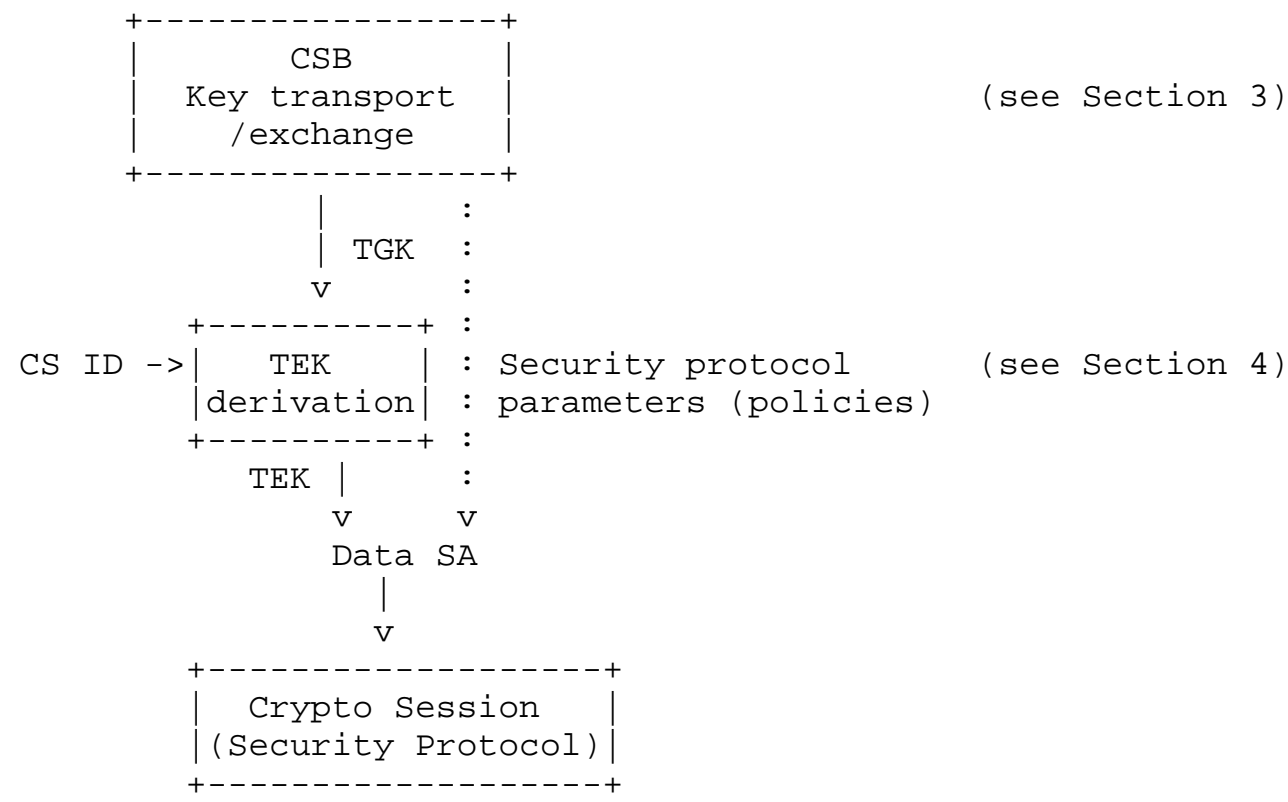

Figure 2.2: Overview of MIKEY key management procedure.

The security protocol can then either use the TEK directly, or, if supported, derive further session keys from the TEK (e.g., see SRTP [SRTP]). It is however up to the security protocol to define how the TEK is used.

MIKEY can be used to update TEKs and the Crypto Sessions in a current Crypto Session Bundle (see Section 4.5). This is done by executing the transport/exchange phase once again to obtain a new TGK (and consequently derive new TEKS) or to update some other specific CS parameters. 


\subsection{Relation to GKMARCH}

The Group key management architecture (GKMARCH) [GKMARCH] describes a general architecture for group key management protocols. MIKEY is a part of this architecture, and can be used as a so-called Registration protocol. The main entities involved in the architecture are the group controller/key server (GCKS), the receiver $(s)$, and the sender $(s)$.

In MIKEY, the sender could act as GCKS and push keys down to the receiver $(s)$.

Note that, for example, in a SIP-initiated call, the sender may also be a receiver. As MIKEY addresses small interactive groups, a member may dynamically change between being a sender and receiver (or being both simultaneously).

\section{Basic Key Transport and Exchange Methods}

The following sub-sections define three different methods of transporting/establishing a TGK: with the use of a pre-shared key, public-key encryption, and Diffie-Hellman (DH) key exchange. In the following, we assume unicast communication for simplicity. In addition to the TGK, a random "nonce", denoted RAND, is also transported. In all three cases, the TGK and RAND values are then used to derive TEKs as described in Section 4.1.3. A timestamp is also sent to avoid replay attacks (see section 5.4).

The pre-shared key method and the public-key method are both based on key transport mechanisms, where the actual TGK is pushed (securely) to the recipient(s). In the Diffie-Hellman method, the actual TGK is instead derived from the Diffie-Hellman values exchanged between the peers.

The pre-shared case is, by far, the most efficient way to handle the key transport due to the use of symmetric cryptography only. This approach also has the advantage that only a small amount of data has to be exchanged. Of course, the problematic issue is scalability as it is not always feasible to share individual keys with a large group of peers. Therefore, this case mainly addresses scenarios such as server-to-client and also those cases where the public-key modes have already been used, thus allowing for the "cache" of a symmetric key (see below and section 3.2).

Public-key cryptography can be used to create a scalable system. A disadvantage with this approach is that it is more resource consuming than the pre-shared key approach. Another disadvantage is that in most cases, a PKI (Public Key Infrastructure) is needed to handle the 
distribution of public keys. Of course, it is possible to use public keys as pre-shared keys (e.g., by using self-signed certificates). It should also be noted that, as mentioned above, this method may be used to establish a "cached" symmetric key that later can be used to establish subsequent TGKs by using the pre-shared key method (hence, the subsequent request can be executed more efficiently).

In general, the Diffie-Hellman (DH) key agreement method has a higher resource consumption (both computationally and in bandwidth) than the previous ones, and needs certificates as in the public-key case. However, it has the advantage of providing perfect forward secrecy (PFS) and flexibility by allowing implementation in several different finite groups.

Note that by using the DH method, the two involved parties will generate a unique unpredictable random key. Therefore, it is not possible to use this DH method to establish a group TEK (as the different parties in the group would end up with different TEKs). It is not the intention of the $\mathrm{DH}$ method to work in this scenario, but to be a good alternative in the special peer-to-peer case.

The following general notation is used:

HDR: The general MIKEY header, which includes MIKEY CSB related data (e.g., CSB ID) and information mapping to the specific security protocol used. See section 6.1 for payload definition.

T: The timestamp, used mainly to prevent replay attacks. See Section 6.6 for payload definition and also section 5.4 for other timestamp related information.

IDx: The identity of entity x (IDi=Initiator, IDr=Responder). See Section 6.7 for payload definition.

RAND: Random/pseudo-random byte-string, which is always included in the first message from the Initiator. RAND is used as a freshness value for the key generation. It is not included in update messages of a CSB. See Section 6.11 for payload definition. For randomness recommendations for security, see [RAND].

SP: The security policies for the data security protocol. See Section 6.10 for payload definition. 


\subsection{Pre-shared key}

In this method, the pre-shared secret key, s, is used to derive key material for both the encryption (encr_key) and the integrity protection (auth_key) of the MIKEY messages, as described in section 4.1.4. The encryption and authentication transforms are described in Section 4.2 .

Initiator

I_MESSAGE =

HDR, T, RAND, [IDi], [IDr], $\{\mathrm{SP}\}, \mathrm{KEMAC}$
Responder

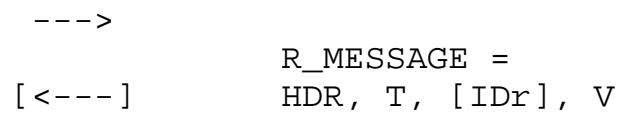

The main objective of the Initiator's message (I_MESSAGE) is to transport one or more TGKs (carried into KEMAC) and a set of security parameters (SPS) to the Responder in a secure manner. As the verification message from the Responder is optional, the Initiator indicates in the HDR whether it requires a verification message or not from the Responder.

$\mathrm{KEMAC}=\mathrm{E}($ encr_key, $\{\mathrm{TGK}\})|| \mathrm{MAC}$

The KEMAC payload contains a set of encrypted sub-payloads and a MAC. Each sub-payload includes a TGK randomly and independently chosen by the Initiator (and other possible related parameters, e.g., the key lifetime). The MAC is a Message Authentication Code covering the entire MIKEY message using the authentication key, auth_key. See Section 6.2 for payload definition and section 5.2 for an exact definition of the MAC calculation.

The main objective of the verification message from the Responder is to obtain mutual authentication. The verification message, $V$, is a MAC computed over the Responder's entire message, the timestamp (the same as the one that was included in the Initiator's message), and the two parties identities, using the authentication key. See also Section 5.2 for the exact definition of the Verification MAC calculation and section 6.9 for payload definition.

The ID fields SHOULD be included, but they MAY be left out when it can be expected that the peer already knows the other party's ID (otherwise it cannot look up the pre-shared key). For example, this could be the case if the ID is extracted from SIP.

It is MANDATORY to implement this method. 
3.2. Public-key encryption

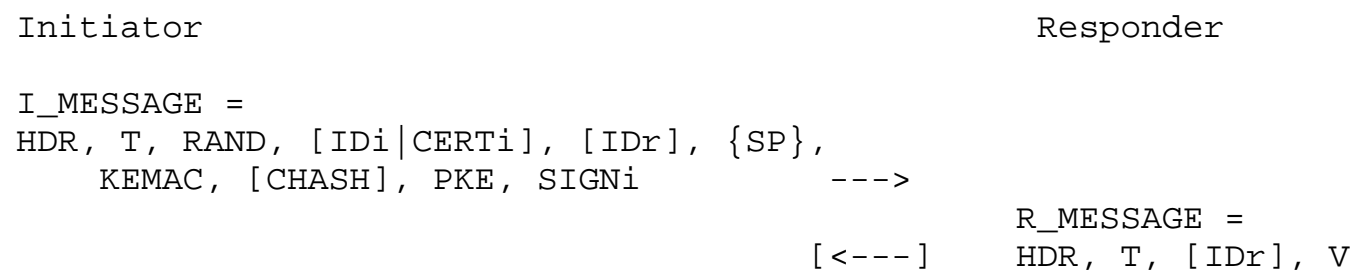


Note that there will be one encrypted IDi and possibly also one unencrypted IDi. The encrypted one is used together with the MAC as a countermeasure for certain man-in-the-middle attacks, while the unencrypted one is always useful for the Responder to immediately identify the Initiator. The encrypted IDi MUST always be verified to be equal with the expected IDi.

It is possible to cache the envelope key, so that it can be used as a pre-shared key. It is not recommended for this key to be cached indefinitely (however it is up to the local policy to decide this). This function may be very convenient during the lifetime of a CSB, if a new crypto session needs to be added (or an expired one removed). Then, the pre-shared key can be used, instead of the public keys (see also Section 4.5). If the Initiator indicates that the envelope key should be cached, the key is at least to be cached during the lifetime of the entire CSB.

The cleartext ID fields and certificate SHOULD be included, but they MAY be left out when it can be expected that the peer already knows the other party's ID, or can obtain the certificate in some other manner. For example, this could be the case if the ID is extracted from SIP.

For certificate handling, authorization, and policies, see section 4.3 .

It is MANDATORY to implement this method.

\subsection{Diffie-Hellman key exchange}

For a fixed, agreed upon, cyclic group, (G,*), we let $g$ denote a generator for this group. Choices for the parameters are given in Section 4.2.7. The other transforms below are described in section 4.2 .

This method creates a DH-key, which is used as the TGK. This method cannot be used to create group keys; it can only be used to create single peer-to-peer keys. It is OPTIONAL to implement this method.

Initiator

I_MESSAGE =

HDR, T, RAND, [IDi|CERTi], [IDr]

$\{\mathrm{SP}\}, \mathrm{DH} i, \mathrm{SIGNi}$
Responder

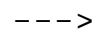

R_MESSAGE =

HDR, T, [IDr|CERTr], IDi, DHr, DHi, SIGNr 
The main objective of the Initiator's message is to, in a secure way, provide the Responder with its $\mathrm{DH}$ value (DHi) $\mathrm{g}^{\wedge}(\mathrm{xi})$, where $\mathrm{xi}$ MUST be randomly/pseudo-randomly and secretly chosen, and a set of security protocol parameters.

The SIGNi is a signature covering the Initiator's MIKEY message, I_MESSAGE, using the Initiator's signature key (see section 5.2 for the exact definition).

The main objective of the Responder's message is to, in a secure way, provide the Initiator with the Responder's value (DHr) $g^{\wedge}(\mathrm{xr})$, where xr MUST be randomly/pseudo-randomly and secretly chosen. The timestamp that is included in the answer is the same as the one included in the Initiator's message.

The SIGNr is a signature covering the Responder's MIKEY message, R_MESSAGE, using the Responder's signature key (see Section 5.2 for the exact definition).

The DH group parameters (e.g., the group G, the generator g) are chosen by the Initiator and signaled to the Responder. Both parties calculate the TGK, $g^{\wedge}(x i * x r)$ from the exchanged DH-values.

Note that this approach does not require that the Initiator has to possess any of the Responder's certificates before the setup. Instead, it is sufficient that the Responder includes its signing certificate in the response.

The ID fields and certificate SHOULD be included, but they MAY be left out when it can be expected that the peer already knows the other party's ID (or can obtain the certificate in some other manner). For example, this could be the case if the ID is extracted from SIP.

For certificate handling, authorization, and policies, see section 4.3 .

4. Selected Key Management Functions

MIKEY manages symmetric keys in two main ways. First, following key transport or key exchange of TGK(s) (and other parameters) as defined by any of the above three methods, MIKEY maintains a mapping between Data SA identifiers and Data SAs, where the identifiers used depend on the security protocol in question, see section 4.4. Thus, when the security protocol requests a Data SA, given such a Data SA identifier, an up-to-date Data SA will be obtained. In particular, 
correct keying material, TEK(s), might need to be derived. The derivation of TEK(s) (and other keying material) is done from a TGK and is described in section 4.1.3.

Second, for use within MIKEY itself, two key management procedures are needed:

* in the pre-shared case, deriving encryption and authentication key material from a single pre-shared key, and

* in the public key case, deriving similar key material from the transported envelope key.

These two key derivation methods are specified in section 4.1.4.

All the key derivation functionality mentioned above is based on a pseudo-random function, defined next.

4.1. Key Calculation

In the following, we define a general method (pseudo-random function)

to derive one or more keys from a "master" key. This method is used

to derive:

* TEKs from a TGK and the RAND value,

* encryption, authentication, or salting key from a pre-shared/ envelope key and the RAND value.

\subsubsection{Assumptions}

We assume that the following parameters are in place:

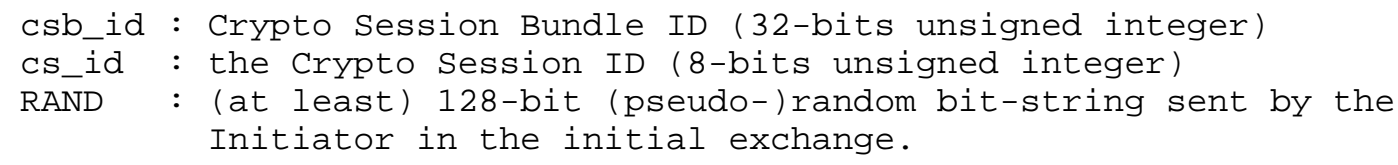

The key derivation method has the following input parameters:

inkey : the input key to the derivation function

inkey_len : the length in bits of the input key

label : a specific label, dependent on the type of the key to be derived, the RAND, and the session IDs

outkey_len: desired length in bits of the output key. 
The key derivation method has the following output:

outkey: the output key of desired length.

\subsubsection{Default PRF Description}

Let HMAC be the SHA-1 based message authentication function, see [HMAC] [SHA-1]. Similarly to [TLS], we define:

$$
\begin{aligned}
& \mathrm{P}(\mathrm{s}, \mathrm{label}, \mathrm{m})=\underset{\operatorname{HMAC}}{\operatorname{HMAC}(\mathrm{s}, \mathrm{A}, \mathrm{A}, 2}\left|\begin{array}{l}
\text { label }) \\
\text { label })
\end{array}\right| \mid \ldots \\
& \operatorname{HMAC}\left(\mathrm{s}, \mathrm{A} \_\mathrm{m}\right. \text { label) }
\end{aligned}
$$

where

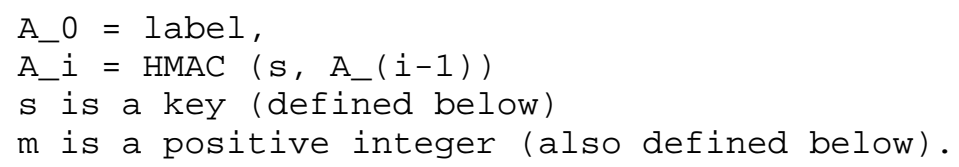




\subsubsection{Generating keys from TGK}

In the following, we describe how keying material is derived from a TGK, thus assuming that a mapping of the Data SA identifier to the correct TGK has already been done according to section 4.4.

The key derivation method SHALL be executed using the above PRF with the following input parameters:

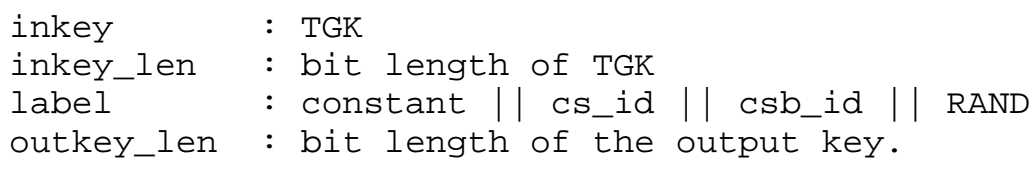

The constant part of label depends on the type of key that is to be generated. The constant 0x2AD01C64 is used to generate a TEK from TGK. If the security protocol itself does not support key derivation for authentication and encryption from the TEK, separate authentication and encryption keys MAY be created directly for the security protocol by replacing 0x2AD01C64 with 0x1B5C7973 and 0x15798CEF respectively, and outkey_len by the desired key-length(s) in each case.

A salt key can be derived from the TGK as well, by using the constant 0x39A2C14B. Note that the Key data sub-payload (Section 6.13) can carry a salt. The security protocol in need of the salt key SHALL use the salt key carried in the Key data sub-payload (in the preshared and public-key case), when present. If that is not sent, then it is possible to derive the salt key via the key derivation function, as described above.

The table below summarizes the constant values, used to generate keys from a TGK.

constant | derived key from the TGK

\begin{tabular}{l|l}
$0 \times 2 A D 01 C 64$ & TEK \\
$0 \times 1 B 5 C 7973$ & $\begin{array}{l}\text { authentication key } \\
\text { encryption key } \\
\text { 0x15798CEF } \\
\text { 0x39A2C14B }\end{array}$
\end{tabular}

Table 4.1.3: Constant values for the derivation of keys from TGK.

Note that these 32-bit constant values (listed in the table above) are taken from the decimal digits of e (i.e., 2.7182...), where each constant consists of nine decimal digits (e.g., the first nine decimal digits $718281828=0 \times 2 A D 01 C 64)$. The strings of nine 
decimal digits are not chosen at random, but as consecutive "chunks" from the decimal digits of e.

4.1.4. Generating keys for MIKEY messages from an envelope/pre-shared key

This derivation is to form the symmetric encryption key (and salting key) for the encryption of the TGK in the pre-shared key and public key methods. This is also used to derive the symmetric key used for the message authentication code in these messages, and the corresponding verification messages. Hence, this derivation is needed in order to get different keys for the encryption and the MAC (and in the case of the pre-shared key, it will result in fresh key material for each new CSB). The parameters for the default PRF are here:

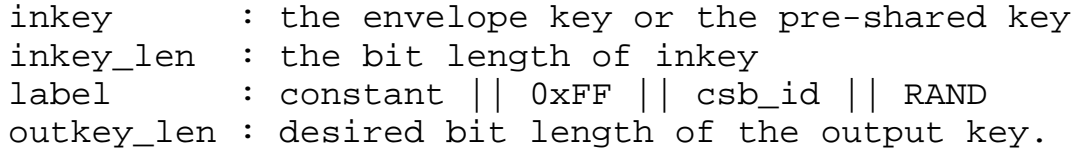

Table 4.1.4: Constant values for the derivation of keys from an envelope/pre-shared key.

\subsection{Pre-defined Transforms and Timestamp Formats}

This section identifies default transforms for MIKEY. It is mandatory to implement and support the following transforms in the respective case. New transforms can be added in the future (see Section 4.2.9 for further guidelines).

\subsubsection{Hash functions}

In MIKEY, it is MANDATORY to implement SHA-1 as the default hash function. 


\subsubsection{Pseudo-random number generator and PRF}

A cryptographically secure random or pseudo-random number generator MUST be used for the generation of the keying material and nonces, e.g., [BMGL]. However, which one to use is implementation specific (as the choice will not affect the interoperability).

For the key derivations, it is MANDATORY to implement the PRF specified in Section 4.1. Other PRFS MAY be added by writing standard-track RFCs specifying the PRF constructions and their exact use within MIKEY.

\subsubsection{Key data transport encryption}

The default and mandatory-to-implement key transport encryption is AES in counter mode, as defined in [SRTP], using a 128-bit key as derived in Section 4.1.4, SRTP_PREFIX_LENGTH set to zero, and using the initialization vector

$I V=(S \mathrm{XOR}(0 \times 0000\|\mathrm{CSB} I D\| \mathrm{T})) \| 0 \times 0000$

where $S$ is a 112-bit salting key, also derived as in section 4.1.4, and where $\mathrm{T}$ is the 64-bit timestamp sent by the Initiator.

Note: this restricts the maximum size that can be encrypted to $2 \wedge 23$ bits, which is still enough for all practical purposes [SRTP].

The NULL encryption algorithm (i.e., no encryption) can be used (but implementation is OPTIONAL). Note that this MUST NOT be used unless the underlying protocols can guarantee security. The main reason for including this is for specific SIP scenarios, where SDP is protected end-to-end. For this scenario, MIKEY MAY be used with the pre-shared key method, the NULL encryption, and NULL authentication algorithm (see section 4.2.4) while relying on the security of SIP. Use this option with caution!

The AES key wrap function [AESKW] is included as an OPTIONAL implementation method. If the key wrap function is used in the public key method, the NULL MAC is RECOMMENDED to be used, as the key wrap itself will provide integrity of the encrypted content (note though that the NULL MAC SHOULD NOT be used in the pre-shared key case, as the MAC in that case covers the entire message). The 128bit key and a 64-bit salt, $\mathrm{S}$, are derived in accordance to section 4.1.4 and the key wrap IV is then set to $\mathrm{S}$. 


\subsubsection{MAC and Verification Message function}

MIKEY uses a 160-bit authentication tag, generated by HMAC with SHA-1 as the MANDATORY implementation method, see [HMAC]. Authentication keys are derived according to Section 4.1.4. Note that the authentication key size SHOULD be equal to the size of the hash function's output (e.g., for HMAC-SHA-1, a 160-bit authentication key is used) [HMAC].

The NULL authentication algorithm (i.e., no MAC) can be used together with the NULL encryption algorithm (but implementation is OPTIONAL). Note that this MUST NOT be used unless the underlying protocols can guarantee security. The main reason for including this is for specific SIP scenarios, where SDP is protected end-to-end. For this scenario, MIKEY MAY be used with the pre-shared key method and the NULL encryption and authentication algorithm, while relying on the security of SIP. Use this option with caution!

4.2.5. Envelope Key encryption

The public key encryption algorithm applied is defined by, and dependent on the certificate used. It is MANDATORY to support RSA PKCS\#1, v1.5, and it is RECOMMENDED to also support RSA OAEP [PSS].

\subsubsection{Digital Signatures}

The signature algorithm applied is defined by, and dependent on the certificate used. It is MANDATORY to support RSA PKCS\#1, v1.5, and it is RECOMMENDED to also support RSA PSS [PSS].

\subsubsection{Diffie-Hellman Groups}

The Diffie-Hellman key exchange, when supported, uses OAKLEY 5 [OAKLEY] as a mandatory implementation. Both OAKLEY 1 and OAKLEY 2 MAY be used (but these are OPTIONAL implementations).

See Section 4.2.9 for the guidelines on specifying a new DH Group to be used within MIKEY.

\subsubsection{Timestamps}

The timestamp is as defined in NTP [NTP], i.e., a 64-bit number in seconds relative to $0 h$ on 1 January 1900. An implementation MUST be aware of (and take into account) the fact that the counter will overflow approximately every 136th year. It is RECOMMENDED that the time always be specified in UTC. 


\subsubsection{Adding new parameters to MIKEY}

There are two different parameter sets that can be added to MIKEY. The first is a set of MIKEY transforms (needed for the exchange itself), and the second is the Data SAs.

New transforms and parameters (including new policies) SHALL be added by registering with IANA (according to [RFC2434], see also section 10) a new number for the concerned payload, and also if necessary, documenting how the new transform/parameter is used. Sometimes it might be enough to point to an already specified document for the usage, e.g., when adding a new, already standardized, hash function.

In the case of adding a new DH group, the group MUST be specified in a companion standards-track RFC (it is RECOMMENDED that the specified group use the same format as used in [OAKLEY]). A number can then be assigned by IANA for such a group to be used in MIKEY.

When adding support for a new data security protocol, the following MUST be specified:

* A map sub-payload (see Section 6.1). This is used to be able to map a crypto session to the right instance of the data security protocol and possibly also to provide individual parameters for each data security protocol.

* A policy payload, i.e., specification of parameters and supported values.

* General guidelines of usage.

4.3. Certificates, Policies and Authorization

\subsubsection{Certificate handling}

Certificate handling may involve a number of additional tasks not shown here, and effect the inclusion of certain parts of the message (c.f. [X.509]). However, the following observations can be made:

* The Initiator typically has to find the certificate of the Responder in order to send the first message. If the Initiator does not already have the Responder's certificate, this may involve one or more roundtrips to a central directory agent.

* It will be possible for the Initiator to omit its own certificate and rely on the Responder getting this certificate using other means. However, we only recommend doing this when it is reasonable to expect that the Responder has cached the certificate 
from a previous connection. Otherwise accessing the certificate would mean additional roundtrips for the Responder as well.

* Verification of the certificates using Certificate Revocation Lists (CRLS) [X.509] or protocols such as OCSP [OCSP] may be necessary. All parties in a MIKEY exchange should have a local policy which dictates whether such checks are made, how they are made, and how often they are made. Note that performing the checks may imply additional messaging.

\subsubsection{Authorization}

In general, there are two different models for making authorization decisions for both the Initiator and the Responder, in the context of the applications targeted by MIKEY:

* Specific peer-to-peer configuration. The user has configured the application to trust a specific peer.

When pre-shared secrets are used, this is pretty much the only available scheme. Typically, the configuration/entering of the pre-shared secret is taken to mean that authorization is implied.

In some cases, one could also use this with public keys, e.g., if two peers exchange keys offline and configure them to be used for the purpose of running MIKEY.

* Trusted root. The user accepts all peers that prove to have a certificate issued by a specific CA. The granularity of authorization decisions is not very precise in this method.

In order to make this method possible, all participants in the MIKEY protocol need to configure one or more trusted roots. The participants also need to be capable of performing certificate chain validation, and possibly transfer more than a single certificate in the MIKEY messages (see also section 6.7).

In practice, a combination of both mentioned methods might be advantageous. Also, the possibility for a user to explicitly exclude a specific peer (or sub-tree) in a trust chain might be needed.

These authorization policies address the MIKEY scenarios a-c of Section 2.1, where the Initiator acts as the group owner and is also the only one that can invite others. This implies that for each Responder, the distributed keys MUST NOT be re-distributed to other parties. 
In a many-to-many situation, where the group control functions are distributed (and/or where it is possible to delegate the group control function to others), a means of distributing authorization information about who may be added to the group MUST exist. However, it is out of scope of this document to specify how this should be done.

For any broader communication situation, an external authorization infrastructure may be used (following the assumptions of [GKMARCH]).

\subsubsection{Data Policies}

Included in the message exchange, policies (i.e., security parameters) for the Data security protocol are transmitted. The policies are defined in a separate payload and are specific to the security protocol (see also Section 6.10). Together with the keys, the validity period of these can also be specified. For example, this can be done with an SPI (or SRTP MKI) or with an Interval (e.g., a sequence number interval for SRTP), depending on the security protocol.

New parameters can be added to a policy by documenting how they should be interpreted by MIKEY and by also registering new values in the appropriate name space in IANA. If a completely new policy is needed, see Section 4.2.9 for guidelines.

\subsection{Retrieving the Data SA}

The retrieval of a Data SA will depend on the security protocol, as different security protocols will have different characteristics. When adding support for a security protocol to MIKEY, some interface of how the security protocol retrieves the Data SA from MIKEY MUST be specified (together with policies that can be negotiated).

For SRTP, the SSRC (see [SRTP]) is one of the parameters used to retrieve the Data SA (while the MKI may be used to indicate the TGK/TEK used for the Data SA). However, the SSRC is not sufficient. For the retrieval of the Data SA from MIKEY, it is RECOMMENDED that the MIKEY implementation support a lookup using destination network address and port together with SSRC. Note that MIKEY does not send network addresses or ports. One reason for this is that they may not be known in advance. Also, if a NAT exists in-between, problems may arise. When SIP or RTSP is used, the local view of the destination address and port can be obtained from either SIP or RTSP. MIKEY Can then use these addresses as the index for the Data SA lookup. 


\subsection{TGK re-keying and CSB updating}

MIKEY provides a means of updating the CSB (e.g., transporting a new TGK/TEK or adding a new Crypto session to the CSB). The updating of the CSB is done by executing MIKEY again, for example, before a TEK expires, or when a new Crypto Session is added to the CSB. Note that MIKEY does not provide re-keying in the GKMARCH sense, only updating of the keys by normal unicast messages.

When MIKEY is executed again to update the CSB, it is not necessary to include certificates and other information that was provided in the first exchange, for example, all payloads that are static or optionally included may be left out (see Figure 4.1).

The new message exchange MUST use the same CSB ID as the initial exchange, but MUST use a new timestamp. A new RAND MUST NOT be included in the message exchange (the RAND will only have effect in the Initial exchange). If desired, new Crypto sessions are added in the update message. Note that a MIKEY update message does not need to contain new keying material (e.g., new TGK). In this case, the crypto session continues to use the previously established keying material, while updating the new information.

As explained in Section 3.2, the envelope key can be "cached" as a pre-shared key (this is indicated by the Initiator in the first message sent). If so, the update message is a pre-shared key message with the cached envelope key as the pre-shared key; it MUST NOT be a public key message. If the public key message is used, but the envelope key is not cached, the Initiator MUST provide a new encrypted envelope key that can be used in the verification message. However, the Initiator does not need to provide any other keys.

Figure 4.1 visualizes the update messages that can be sent, including the optional parts. The main difference from the original message is that it is optional to include TGKS (or DH values in the DH method). Also see Section 3 for more details on the specific methods.

By definition, a CSB can contain several CSs. A problem that then might occur is to synchronize the TGK re-keying if an SPI (or similar functionality, e.g., MKI in [SRTP]) is not used. It is therefore RECOMMENDED that an SPI or MKI be used, if more than one CS is present. 
choose a common set of parameters. This is done by including one or more security policy payloads in the error message sent in response (see section 5.1.2.). Multiple attributes can be provided in sequence in the response. This is done to reduce the number of roundtrips as much as possible (i.e., in most cases, where the policy is accepted the first time, one roundtrip is enough). If the Responder does not accept the offer, the Initiator must go out with a new MIKEY message.

If the Responder is not willing/capable of providing security or the parties simply cannot agree, it is up to the parties' policies how to behave, for example, accepting or rejecting an insecure communication.

Note that it is not the intention of this protocol to have a broad variety of options, as it is assumed that a denied offer should rarely occur.

In the one-to-many and many-to-many scenarios using multicast communication, one issue is of course that there MUST be a common security policy for all the receivers. This limits the possibility of negotiation.

\subsubsection{Error Handling}

Due to the key management protocol, all errors SHouLD be reported to the peer(s) by an error message. The Initiator SHould therefore always be prepared to receive such a message from the Responder.

If the Responder does not support the set of parameters suggested by the Initiator, the error message SHOULD include the supported parameters (see also Section 5.1.1).

The error message is formed as:

$\mathrm{HDR}, \mathrm{T},\{\mathrm{ERR}\},\{\mathrm{SP}\},[\mathrm{V} \mid \mathrm{SIGN} r]$

Note that if failure is due to the inability to authenticate the peer, the error message is OPTIONAL, and does not need to be authenticated. It is up to local policy to determine how to treat this kind of message. However, if in response to a failed authentication a signed error message is returned, this can be used for Dos purposes (against the Responder). Similarly, an unauthenticated error message could be sent to the Initiator in order to fool the Initiator into tearing down the CSB. It is highly RECOMMENDED that the local policy take this into consideration. Therefore, in case of authentication failure, one recommendation would be not to authenticate such an error message, and when 
receiving an unauthenticated error message view it only as a recommendation of what may have gone wrong.

\subsection{Creating a message}

To create a MIKEY message, a Common Header payload is first created. This payload is then followed, depending on the message type, by a set of information payloads (e.g., DH-value payload, Signature payload, Security Policy payload). The defined payloads and the exact encoding of each payload are described in section 6 .

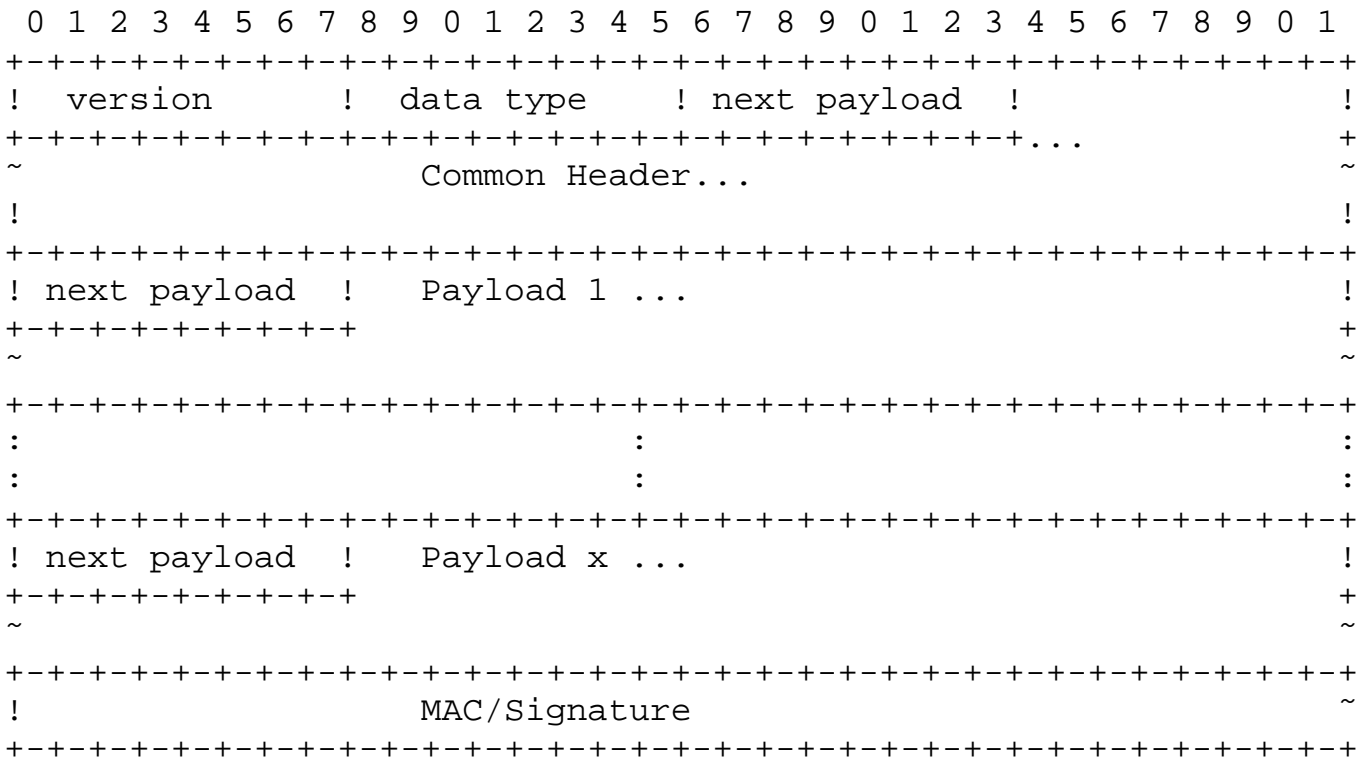

Figure 5.1. MIKEY payload message example. Note that the payloads are byte aligned and not 32-bit aligned.

The process of generating a MIKEY message consists of the following steps:

* Create an initial MIKEY message starting with the Common Header payload.

* Concatenate necessary payloads of the MIKEY message (see the exchange definitions for payloads that may be included, and the recommended order).

* As a last step (for messages that must be authenticated, this also includes the verification message), create and concatenate the MAC/signature payload without the MAC/signature field filled in 
(if a Next payload field is included in this payload, it is set to Last payload).

* Calculate the MAC/signature over the entire MIKEY message, except the MAC/Signature field, and add the MAC/signature in the field. In the case of the verification message, the Identity_i || Identity_r|| Timestamp MUST directly follow the MIKEY message in the Verification MAC calculation. Note that the added identities and timestamp are identical to those transported in the ID and $\mathrm{T}$ payloads.

In the public key case, the Key data transport payload is generated by concatenating the IDi with the TGKs. This is then encrypted and placed in the data field. The MAC is calculated over the entire Key data transport payload except the MAC field. Before calculating the MAC, the Next payload field is set to zero.

Note that all messages from the Initiator MUST use a unique timestamp. The Responder does not create a new timestamp, but uses the timestamp used by the Initiator.

\subsection{Parsing a message}

In general, parsing of a MIKEY message is done by extracting payload by payload and checking that no errors occur. The exact procedure is implementation specific; however, for the Responder, it is RECOMMENDED that the following procedure be followed:

* Extract the Timestamp and check that it is within the allowable clock skew (if not, discard the message). Also check the replay cache (Section 5.4) so that the message is not replayed (see Section 5.4). If the message is replayed, discard it.

* Extract the ID and authentication algorithm (if not included, assume the default).

* Verify the MAC/signature.

* If the authentication is not successful, an Auth failure Error message MAY be sent to the Initiator. The message is then discarded from further processing. See also section 5.1 .2 for treatment of errors.

* If the authentication is successful, the message is processed and also added to the replay cache; processing is implementation specific. Note also that only successfully authenticated messages are stored in the replay cache. 
* If any unsupported parameters or errors occur during the processing, these MAY be reported to the Initiator by sending an error message. The processing is then aborted. The error message can also include payloads to describe the supported parameters.

* If the processing was successful and in case the Initiator requested it, a verification/response message MAY be created and sent to the Initiator.

5.4. Replay handling and timestamp usage

MIKEY does not use a challenge-response mechanism for replay handling; instead, timestamps are used. This requires that the clocks are synchronized. The required synchronization is dependent on the number of messages that can be cached (note though, that the replay cache only contains messages that have been successfully authenticated). If we could assume an unlimited cache, the terminals would not need to be synchronized at all (as the cache could then contain all previous messages). However, if there are restrictions on the size of the replay cache, the clocks will need to be synchronized to some extent. In short, one can in general say that it is a tradeoff between the size of the replay cache and the required synchronization.

Timestamp usage prevents replay attacks under the following assumptions:

* Each host has a clock which is at least "loosely synchronized" with the clocks of the other hosts.

* If the clocks are to be synchronized over the network, a secure network clock synchronization protocol SHOULD be used, e.g., [ISO3].

* Each Responder utilizes a replay cache in order to remember the successfully authenticated messages presented within an allowable clock skew (which is set by the local policy).

* Replayed and outdated messages, for example, messages that can be found in the replay cache or which have an outdated timestamp are discarded and not processed.

* If the host loses track of the incoming requests (e.g., due to overload), it rejects all incoming requests until the clock skew interval has passed. 
In a client-server scenario, servers may encounter a high workload, especially if a replay cache is necessary. However, servers that assume the role of MIKEY Initiators will not need to manage any significant replay cache as they will refuse all incoming messages that are not a response to a message previously sent by the server.

In general, a client may not expect a very high load of incoming messages and may therefore allow the degree of looseness to be on the order of several minutes to hours. If a (D) DoS attack is launched and the replay cache grows too large, MIKEY MAY dynamically decrease the looseness so that the replay cache becomes manageable. However, note that such (D) Dos attacks can only be performed by peers that can authenticate themselves. Hence, such an attack is very easy to trace and mitigate.

The maximum number of messages that a client will need to cache may vary depending on the capacity of the client itself and the network. The number of expected messages should be taken into account.

For example, assume that we can at most spend $6 \mathrm{kB}$ on a replay cache. Assume further that we need to store 30 bytes for each incoming authenticated message (the hash of the message is 20 bytes). This implies that it is possible to cache approximately 204 messages. If the expected number of messages per minute can be estimated, the clock skew can easily be calculated. For example, in a SIP scenario where the client is expected, in the most extreme case, to receive 10 calls per minute, the clock skew needed is then approximately 20 minutes. In a not so extreme setting, where one could expect an incoming call every 5th minute, this would result in a clock skew on the order of 16.5 hours (approx 1000 minutes).

Consider a very extreme case, where the maximum number of incoming messages are assumed to be on the order of 120 messages per minute, and a requirement that the clock skew is on the order of 10 minutes, a $48 \mathrm{kB}$ replay cache would be required.

Hence, one can note that the required clock skew will depend largely on the setting in which MIKEY is used. One recommendation is to fix a size for the replay cache, allowing the clock skew to be large (the initial clock skew can be set depending on the application in which it is used). As the replay cache grows, the clock skew is decreased depending on the percentage of the used replay cache. Note that this is locally handled, which will not require interaction with the peer (even though it may indirectly effect the peer). However, exactly how to implement such functionality is out of the scope of this document and considered implementation specific. 
In case of a Dos attack, the client will most likely be able to handle the replay cache. A more likely (and serious) Dos attack is a CPU DoS attack where the attacker sends messages to the peer, which then needs to expend resources on verifying the MACs/signatures of the incoming messages.

6. Payload Encoding

This section describes, in detail, all the payloads. For all encoding, network byte order is always used. While defining supported types (e.g., which hash functions are supported) the mandatory-to-implement types are indicated (as Mandatory), as well as the default types (note, default also implies mandatory

implementation). Support for the other types are implicitly assumed to be optional.

In the following, note that the support for SRTP [SRTP] as a security protocol is defined. This will help us better understand the purpose of the different payloads and fields. Other security protocols MAY be specified for use within MIKEY, see Section 10.

In the following, the sign indicates variable length field.

\subsection{Common Header payload (HDR)}

The Common Header payload MUST always be present as the first payload in each message. The Common Header includes a general description of the exchange message.

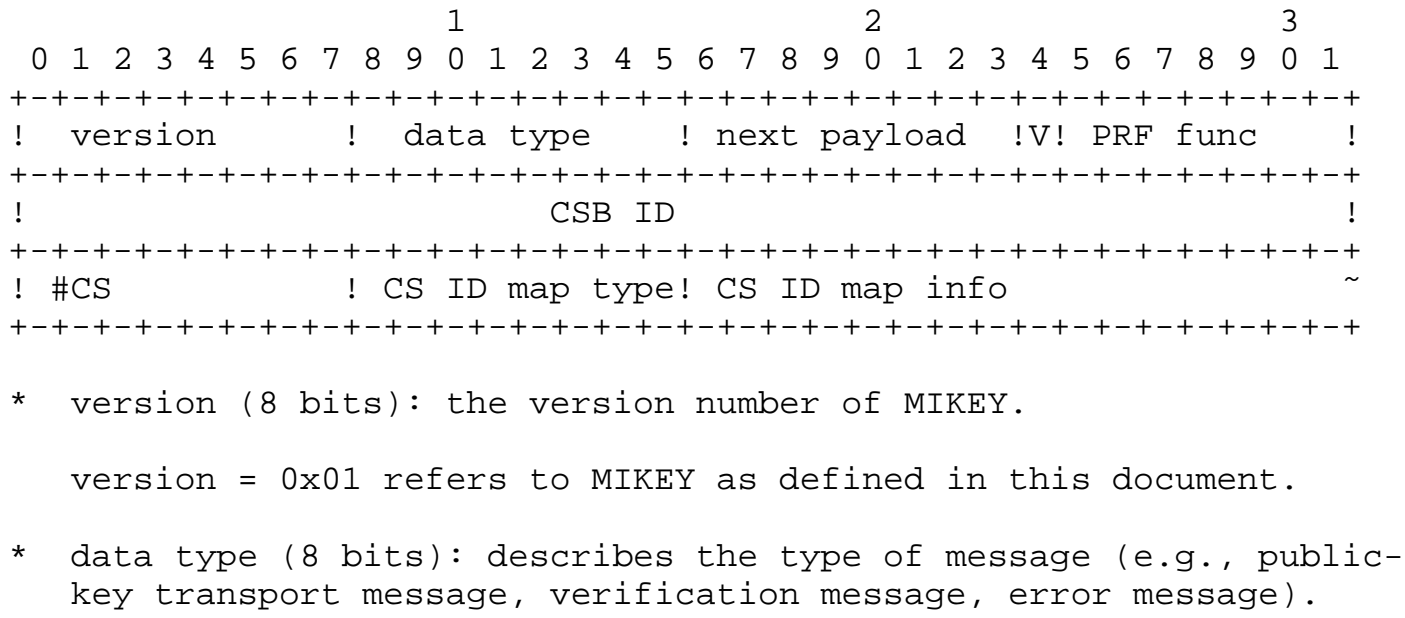




\begin{tabular}{l|l|l} 
Data type & Value & Comment \\
Pre-shared & 0 & Initiator's pre-shared key message \\
PSK ver msg & 1 & $\begin{array}{l}\text { Verification message of a Pre-shared } \\
\text { key message }\end{array}$ \\
$\begin{array}{l}\text { Public key } \\
\text { PK ver msg }\end{array}$ & 2 & $\begin{array}{l}\text { Initiator's public-key transport message } \\
\text { D-H init }\end{array}$ \\
$\begin{array}{l}\text { D-H resp } \\
\text { Error }\end{array}$ & 4 & $\begin{array}{l}\text { Initiator's DH exchange message } \\
\text { message }\end{array}$ \\
\end{tabular}

Table $6.1 . \mathrm{a}$

* next payload (8 bits): identifies the payload that is added after this payload.

Next payload | Value | Section

\begin{tabular}{l|r|l} 
Last payload & 0 & - \\
KEMAC & 1 & 6.2 \\
PKE & 2 & 6.3 \\
DH & 3 & 6.4 \\
SIGN & 4 & 6.5 \\
T & 5 & 6.6 \\
ID & 6 & 6.7 \\
CERT & 7 & 6.7 \\
CHASH & 8 & 6.8 \\
V & 9 & 6.9 \\
SP & 10 & 6.10 \\
RAND & 11 & 6.11 \\
ERR data & 12 & 6.12 \\
Key dat & 20 & 6.13 \\
General Ext. & 21 & 6.15
\end{tabular}

Table $6.1 . \mathrm{b}$

Note that some of the payloads cannot directly follow the header (such as "Last payload", "Signature"). However, the Next payload field is generic for all payloads. Therefore, a value is allocated for each payload. The Next payload field is set to zero (Last payload) if the current payload is the last payload.

* V (1 bit): flag to indicate whether a verification message is expected or not (this only has meaning when it is set by the Initiator). The $\mathrm{V}$ flag SHALL be ignored by the receiver in the $\mathrm{DH}$ method (as the response is MANDATORY). 
$\mathrm{V}=0 \quad==>$ no response expected

$\mathrm{V}=1==>$ response expected

* PRF func (7 bits): indicates the PRF function that has been/will be used for key derivation.

PRF func $\quad$ Value $\mid$ Comments

MIKEY-1 | $0 \mid$ Mandatory (see Section 4.1.2)

Table $6.1 . \mathrm{c}$

* CSB ID (32 bits): identifies the CSB. It is RECOMMENDED that the CSB ID be chosen at random by the Initiator. This ID MUST be unique between each Initiator-Responder pair, i.e., not globally unique. An Initiator MUST check for collisions when choosing the ID (if the Initiator already has one or more established CSBs with the Responder). The Responder uses the same CSB ID in the response.

* \#CS ( 8 bits): indicates the number of Crypto sessions that will be handled within the CBS. Note that even though it is possible to use $255 \mathrm{CSs}$, it is not likely that a CSB will include this many CSs. The integer 0 is interpreted as no CS included. This may be the case in an initial setup message.

* CS ID map type (8 bits): specifies the method of uniquely mapping Crypto Sessions to the security protocol sessions.

CS ID map type | Value

SRTP-ID

Table 6.1.d

* CS ID map info (16 bits): identifies the crypto session(s) for which the SA should be created. The currently defined map type is the SRTP-ID (defined in Section 6.1.1). 
6.1.1. SRTP ID

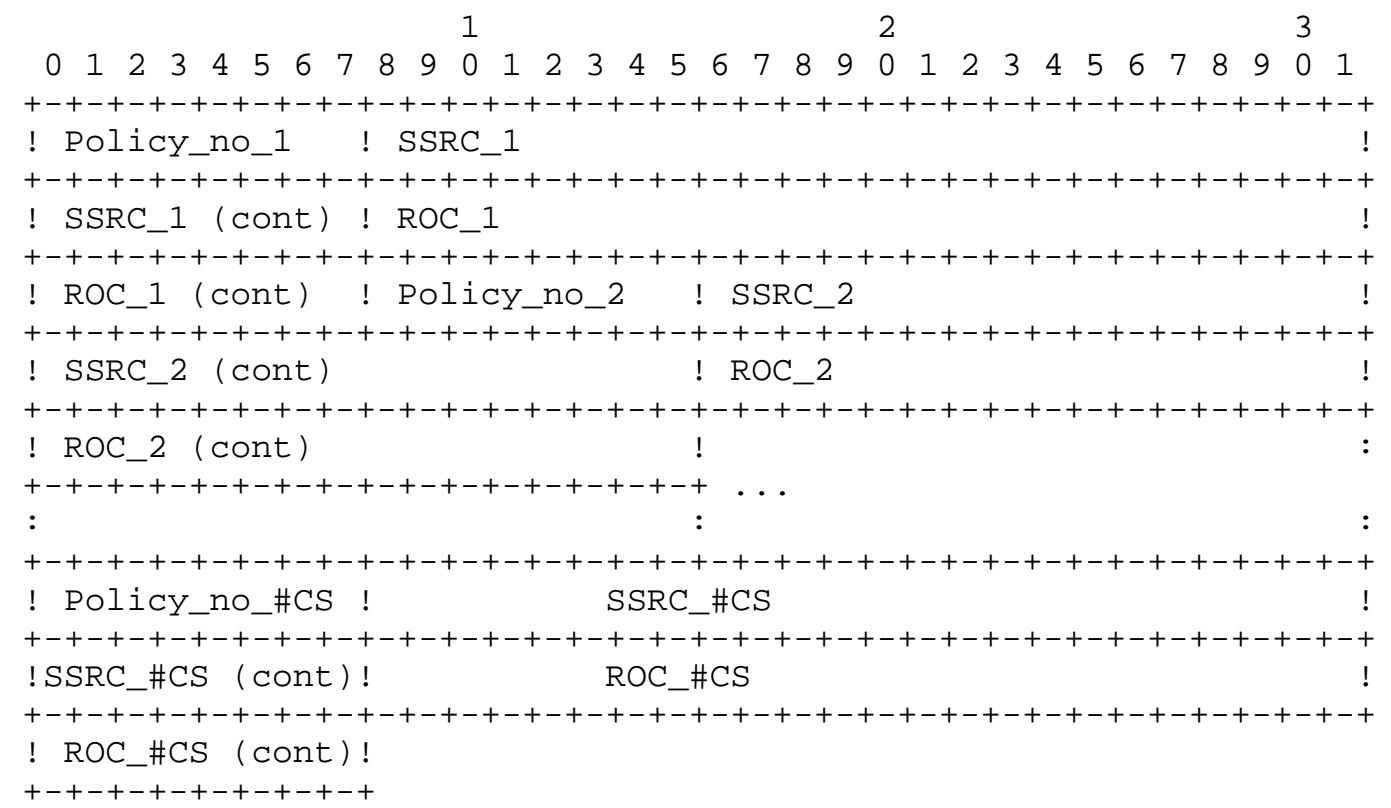

* Policy_no_i (8 bits): The security policy applied for the stream with SSRC_i. The same security policy may apply for all CSs.

* SSRC_i (32 bits): specifies the SSRC that MUST be used for the i-th SRTP stream. Note that it is the sender of the streams that chooses the SSRC. Therefore, it is possible that the Initiator of MIKEY cannot fill in all fields. In this case, SSRCs that are not chosen by the Initiator are set to zero and the Responder fills in these fields in the response message. Note that SRTP specifies requirements on the uniqueness of the SSRCs (to avoid two-time pad problems if the same TEK is used for more than one stream) [SRTP].

* ROC_i (32 bits): Current rollover counter used in SRTP. If the SRTP session has not started, this field is set to 0 . This field is used to enable a member to join and synchronize with an already started stream.

NOTE: The stream using SSRC_i will also have Crypto Session ID equal to no i (NOT to the SSRC). 


\subsection{Key data transport payload (KEMAC)}

The Key data transport payload contains encrypted Key data subpayloads (see section 6.13 for the definition of the Key data subpayload). It may contain one or more key data payloads, each including, for example, a TGK. The last Key data payload has its Next payload field set to Last payload. For an update message (see also Section 4.5), it is allowed to skip the Key data sub-payloads (which will result in the Encr data len being equal to 0 ).

Note that the MAC coverage depends on the method used, i.e., preshared vs public key, see below.

If the transport method used is the pre-shared key method, this key data transport payload is the last payload in the message (note that the Next payload field is set to Last payload). The MAC is then calculated over the entire MIKEY message following the directives in Section 5.2.

If the transport method used is the public-key method, the Initiator's identity is added in the encrypted data. This is done by adding the ID payload as the first payload, which is then followed by the Key data sub-payloads. Note that for an update message, the ID is still sent encrypted to the Responder (this is to avoid certain re-direction attacks) even though no key data sub-payload is added after.

In the public-key case, the coverage of the MAC field is over the Key data transport payload only, instead of the complete MIKEY message, as in the pre-shared case. The MAC is therefore calculated over the Key data transport payload, except for the MAC field and where the Next payload field has been set to zero (see also Section 5.2).

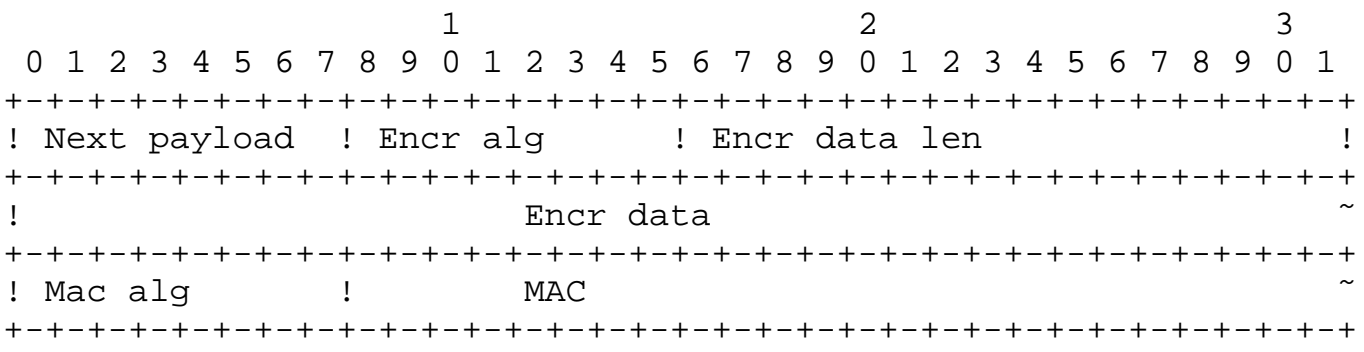

* Next payload ( 8 bits): identifies the payload that is added after this payload. See section 6.1 for defined values.

* Encr alg ( 8 bits): the encryption algorithm used to encrypt the Encr data field. 


\begin{tabular}{|c|c|c|}
\hline Encr alg & Value & Comment \\
\hline NULL & 0 & Very restricted usage, see section 4.2.3! \\
\hline $\mathrm{AES}-\mathrm{CM}-128$ & 1 & $\begin{array}{l}\text { Mandatory; AES-CM using a 128-bit key, see } \\
\text { Section } 4.2 .3 \text { ) }\end{array}$ \\
\hline $\mathrm{AES}-\mathrm{KW}-128$ & 2 & $\begin{array}{l}\text { AES Key Wrap using a 128-bit key, see } \\
\text { Section } 4.2 .3\end{array}$ \\
\hline
\end{tabular}

Table 6.2.a

* Encr data len (16 bits): length of Encr data (in bytes).

* Encr data (variable length): the encrypted key sub-payloads (see Section 6.13).

* MAC alg ( 8 bits): specifies the authentication algorithm used.

\begin{tabular}{l|l|l|l} 
MAC alg & Value & Comments & Length (bits) \\
NULL & 0 & $\begin{array}{l}\text { restricted usage } \\
\text { HMAC-SHA-1-160 }\end{array}$ & $\begin{array}{l}\text { Section 4.2.4 } \\
\text { Mandatory, } \\
\text { Section 4.2.4 }\end{array}$
\end{tabular}

Table $6.2 . \mathrm{b}$

* MAC (variable length): the message authentication code of the entire message.

\subsection{Envelope data payload (PKE)}

The Envelope data payload contains the encrypted envelope key that is used in the public-key transport to protect the data in the Key data transport payload. The encryption algorithm used is implicit from the certificate/public key used.

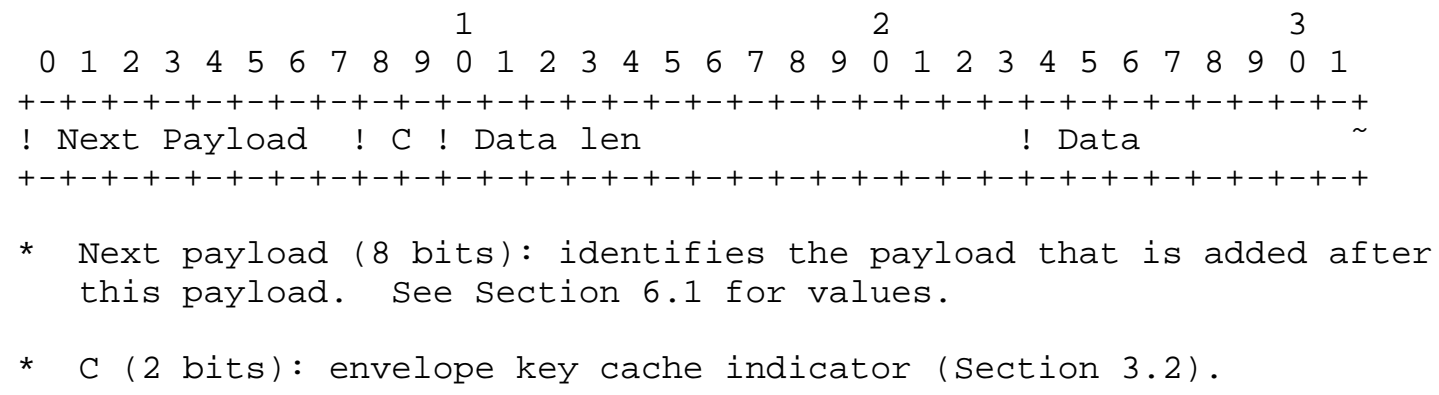




\begin{tabular}{l|l|l} 
Cache type & Value & Comments \\
No cache & 0 & The envelope key MUST NOT be cached \\
Cache & 1 & The envelope key MUST be cached \\
Cache for CSB & 2 & $\begin{array}{l}\text { The envelope key MUST be cached, but only } \\
\text { to be used for the specific CSB. }\end{array}$
\end{tabular}

Table 6.3

* Data len (14 bits): the length of the data field (in bytes).

* Data (variable length): the encrypted envelope key.

6.4. DH data payload (DH)

The DH data payload carries the DH-value and indicates the DH-group used. Notice that in this sub-section, "MANDATORY" is conditioned upon DH being supported.

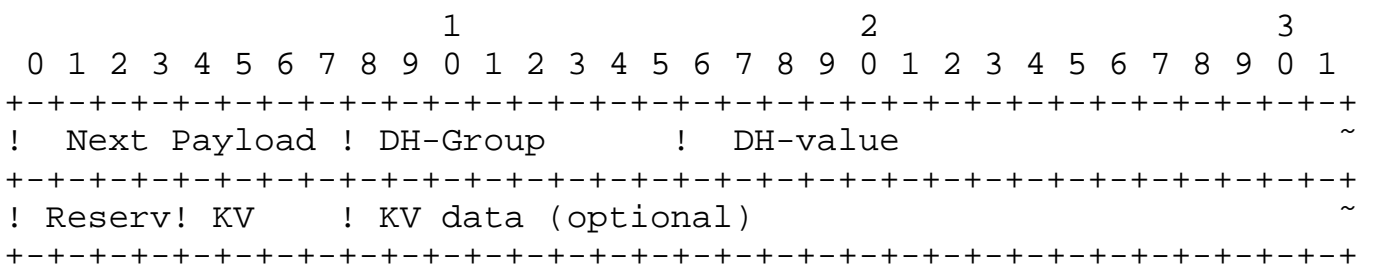

* Next payload ( 8 bits) : identifies the payload that is added after this payload. See section 6.1 for values.

* DH-Group ( 8 bits): identifies the DH group used.

\begin{tabular}{|c|c|c|c|}
\hline DH-Group & Value & Comment & DH Value length (bits) \\
\hline OAKLEY 5 & 0 & Mandatory & 1536 \\
\hline OAKLEY 1 & 1 & & 768 \\
\hline OAKLEY 2 & 2 & & 1024 \\
\hline
\end{tabular}

Table 6.4

* DH-value (variable length): the public DH-value (the length is implicit from the group used).

* KV (4 bits): indicates the type of key validity period specified. This may be done by using an SPI (alternatively an MKI in SRTP) or by providing an interval in which the key is valid (e.g., in the latter case, for SRTP this will be the index range where the key is valid). See section 6.13 for pre-defined values. 
* KV data (variable length): This includes either the SPI/MKI or an interval (see section 6.14). If $\mathrm{KV}$ is NULL, this field is not included.

\subsection{Signature payload (SIGN)}

The Signature payload carries the signature and its related data. The signature payload is always the last payload in the PK transport and $\mathrm{DH}$ exchange messages. The signature algorithm used is implicit from the certificate/public key used.

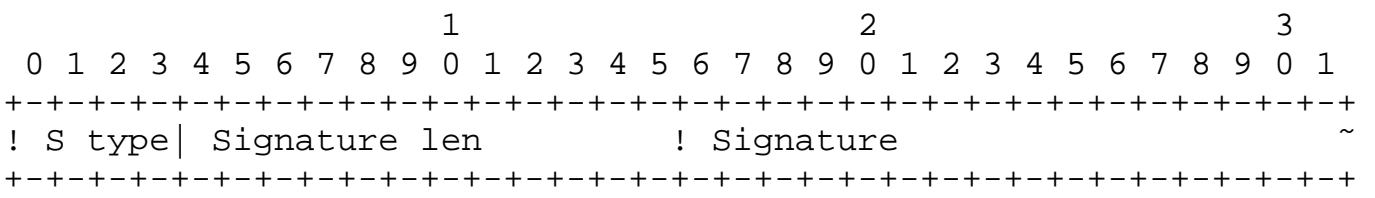

* S type (4 bits): indicates the signature algorithm applied by the signer.

\begin{tabular}{l|l|l} 
S type & Value & Comments \\
- & & \\
RSA/PKCS\#1/1.5 & 0 & Mandatory, PKCS \#1 version 1.5 signature \\
RSA/PSS & 1 & RSASSA-PSS signature [PSS]
\end{tabular}

Table 6.5

* Signature len (12 bits): the length of the signature field (in bytes).

* Signature (variable length): the signature (its formatting and padding depend on the type of signature).

6.6. Timestamp payload (T)

The timestamp payload carries the timestamp information.

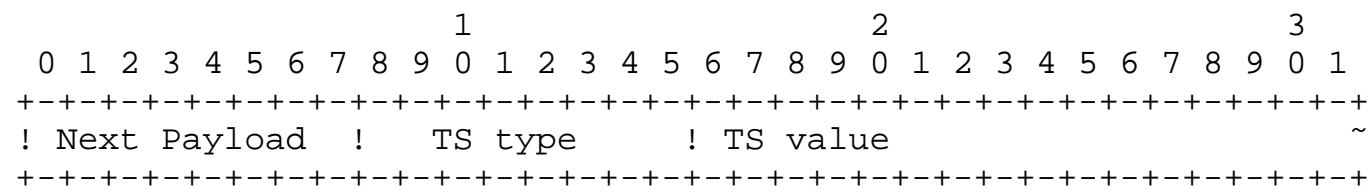

* Next payload ( 8 bits): identifies the payload that is added after this payload. See section 6.1 for values.

* TS type (8 bits): specifies the timestamp type used. 


\begin{tabular}{|c|c|c|c|}
\hline TS type & Value & Comments & length of TS value \\
\hline NTP-UTC & 0 & Mandatory & 64-bits \\
\hline NTP & 1 & Mandatory & 64-bits \\
\hline COUNTER & 2 & Optional & 32-bits \\
\hline
\end{tabular}

Table 6.6

Note: COUNTER SHALL be padded (with leading zeros) to a 64-bit value when used as input for the default PRF.

* TS-value (variable length): The timestamp value of the specified TS type.

6.7. ID payload (ID) / Certificate Payload (CERT)

Note that the ID payload and the Certificate payload are two completely different payloads (having different payload identifiers). However, as they share the same payload structure, they are described in the same section.

The ID payload carries a uniquely defined identifier.

The certificate payload contains an indicator of the certificate provided as well as the certificate data. If a certificate chain is to be provided, each certificate in the chain should be included in a separate CERT payload.

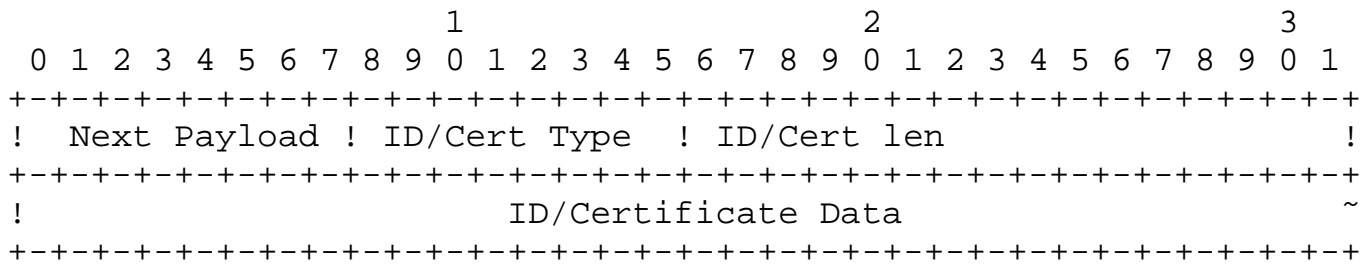

* Next payload ( 8 bits): identifies the payload that is added after this payload. See section 6.1 for values.

If the payload is an ID payload, the following values apply for the ID type field:

* ID Type (8 bits): specifies the identifier type used. 


\begin{tabular}{l|l|l|} 
ID Type & Value & Comments \\
NAI & 0 & Mandatory (see [NAI]) \\
URI & 1 & Mandatory (see [URI])
\end{tabular}

Table 6.7.a

If the payload is a Certificate payload, the following values applies for the Cert type field:

* Cert Type ( 8 bits): specifies the certificate type used.

Cert Type
\begin{tabular}{l|l|l} 
Xalue & Comments \\
X.509v3 & 0 & Mandatory \\
X.509v3 URL & 1 & plain ASCII URL to the location of the Cert \\
X.509v3 Sign & 2 & Mandatory (used for signatures only) \\
X.509v3 Encr & 3 & Mandatory (used for encryption only)
\end{tabular}

Table 6.7.b

* ID/Cert len (16 bits): the length of the ID or Certificate field (in bytes).

* ID/Certificate (variable length): The ID or Certificate data. The X.509 [X.509] certificates are included as a bytes string using DER encoding as specified in X.509.

6.8. Cert hash payload (CHASH)

The Cert hash payload contains the hash of the certificate used.

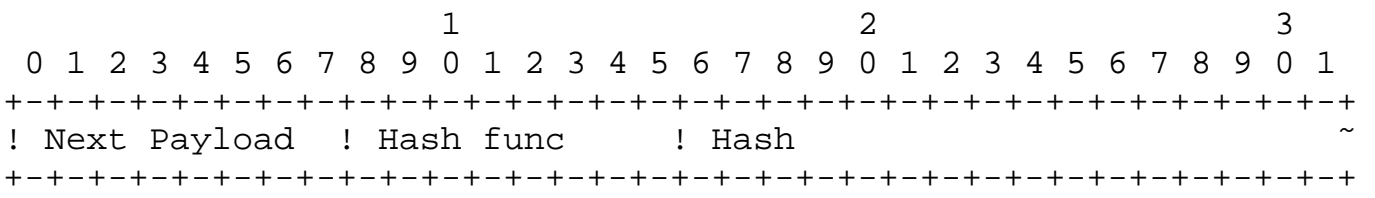

* Next payload (8 bits): identifies the payload that is added after this payload. See Section 6.1 for values.

* Hash func (8 bits): indicates the hash function that is used (see also Section 4.2.1). 


\begin{tabular}{|c|c|c|c|}
\hline Hash func & Value & Comment & hash length \\
\hline $\begin{array}{l}\text { SHA-1 } \\
\text { MD5 }\end{array}$ & $\begin{array}{l}0 \\
1\end{array}$ & Mandatory & $\begin{array}{l}160 \\
128\end{array}$ \\
\hline
\end{tabular}

Table 6.8

* Hash (variable length): the hash data. The hash length is implicit from the hash function used.

6.9. Ver msg payload (V)

The Ver msg payload contains the calculated verification message in the pre-shared key and the public-key transport methods. Note that the MAC is calculated over the entire MIKEY message, as well as the IDs and Timestamp (see also Section 5.2).

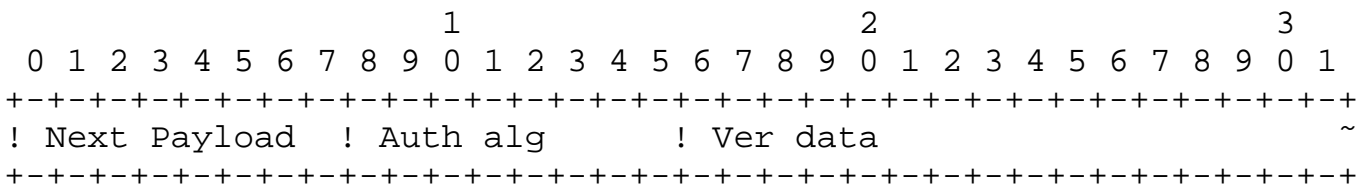

* Next payload ( 8 bits): identifies the payload that is added after this payload. See section 6.1 for values.

* Auth alg ( 8 bits): specifies the MAC algorithm used for the verification message. See Section 6.2 for defined values.

* Ver data (variable length): the verification message data. The length is implicit from the authentication algorithm used.

6.10. Security Policy payload (SP)

The Security Policy payload defines a set of policies that apply to a specific security protocol.

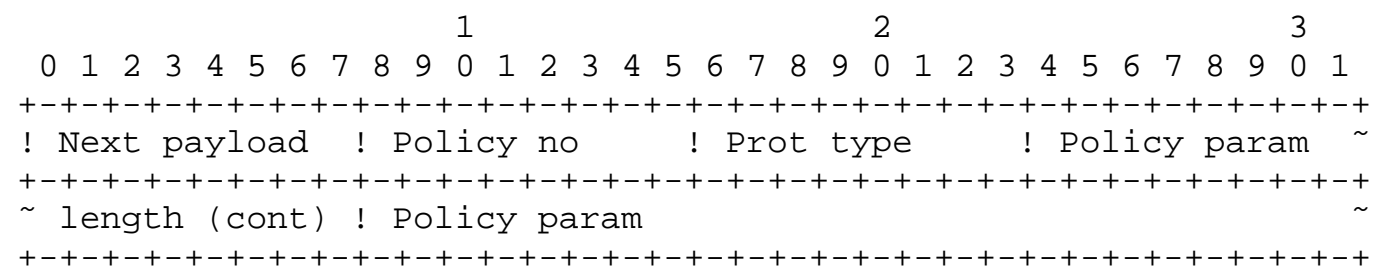

* Next payload ( 8 bits): identifies the payload that is added after this payload. See section 6.1 for values. 
* Policy no ( 8 bits): each security policy payload must be given a distinct number for the current MIKEY session by the local peer. This number is used to map a crypto session to a specific policy (see also Section 6.1.1).

* Prot type (8 bits): defines the security protocol.

\begin{tabular}{l|l} 
Prot type & Value $\mid$ \\
------------ & $\mid$ \\
SRTP & 0
\end{tabular}

Table 6.10

* Policy param length (16 bits): defines the total length of the policy parameters for the specific security protocol.

* Policy param (variable length): defines the policy for the specific security protocol.

The Policy param part is built up by a set of Type/Length/Value fields. For each security protocol, a set of possible types/values that can be negotiated is defined.

$\begin{array}{llllllllllllll}1 & 2 & & \end{array}$

$\begin{array}{llllllllllllllllllllllllllllllll}0 & 1 & 2 & 3 & 4 & 5 & 6 & 7 & 8 & 9 & 0 & 1 & 2 & 3 & 4 & 5 & 6 & 7 & 8 & 9 & 0 & 1 & 2 & 3 & 4 & 5 & 6 & 7 & 8 & 9 & 0 & 1\end{array}$ +-+-+-+-+-+-+-+-+-+-+-+-+-+-+-+-+-+-+-+-+-+-+-+-+-+-+-+-+-+-+-+-+ ! Type ! Length ! Value $+-+-+-+-+-+-+-+-+-+-+-+-+-+-+-+-+-+-+-+-+-+-+-+-+-+-+-+-+-+-+-+-+$

* Type (8 bits): specifies the type of the parameter.

* Length (8 bits): specifies the length of the Value field (in bytes).

* Value (variable length): specifies the value of the parameter. 


\subsubsection{SRTP policy}

This policy specifies the parameters for SRTP and SRTCP. The types/values that can be negotiated are defined by the following table:

\begin{tabular}{|c|c|c|}
\hline Type & Meaning & Possible values \\
\hline 0 & Encryption algorithm & see below \\
\hline 1 & Session Encr. key length & depends on cipher used \\
\hline 2 & Authentication algorithm & see below \\
\hline 3 & Session Auth. key length & depends on MAC used \\
\hline 4 & Session salt key length & see [SRTP] for recommendations \\
\hline 5 & SRTP Pseudo Random Function & see below \\
\hline 6 & Key derivation rate & see [SRTP] for recommendations \\
\hline 7 & SRTP encryption off/on & 0 if off, 1 if on \\
\hline 8 & SRTCP encryption off/on & 0 if off, \\
\hline 9 & sender's FEC order & see below \\
\hline 10 & SRTP authentication off/on & 0 if off, 1 if on \\
\hline 11 & Authentication tag length & in bytes \\
\hline 12 & SRTP prefix length & in bytes \\
\hline
\end{tabular}

Table 6.10.1.a

Note that if a Type/Value is not set, the default is used (according to SRTP's own criteria). Note also that, if "Session Encr. key length" is set, this should also be seen as the Master key length (otherwise, the SRTP default Master key length is used).

For the Encryption algorithm, a one byte length is enough. The currently defined possible values are:

\begin{tabular}{l|r} 
SRTP encr alg & Value \\
\hline NULL & 0 \\
AES-CM & 1 \\
AES-F8 & 2
\end{tabular}

Table 6.10.1.b

where AES-CM is AES in CM, and AES-F 8 is AES in 18 mode [SRTP]. 
For the Authentication algorithm, a one byte length is enough. The currently defined possible Values are:

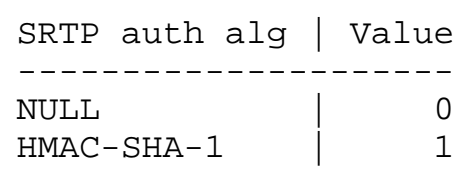

Table 6.10.1.c

For the SRTP pseudo-random function, a one byte length is also enough. The currently defined possible Values are:

\begin{tabular}{l|r} 
SRTP PRF & Value \\
------------- & D
\end{tabular}

Table 6.10.1.d

If FEC is used at the same time SRTP is used, MIKEY can negotiate the order in which these should be applied at the sender side.

\begin{tabular}{l|l|l|} 
FEC order & Value & Comments \\
-------- & \\
FEC-SRTP & 0 & First FEC, then SRTP
\end{tabular}

Table 6.10.1.e

6.11. RAND payload (RAND)

The RAND payload consists of a (pseudo-) random bit-string. The RAND MUST be independently generated per CSB (note that if the CSB has several members, the Initiator MUST use the same RAND for all the members). For randomness recommendations for security, see [RAND].

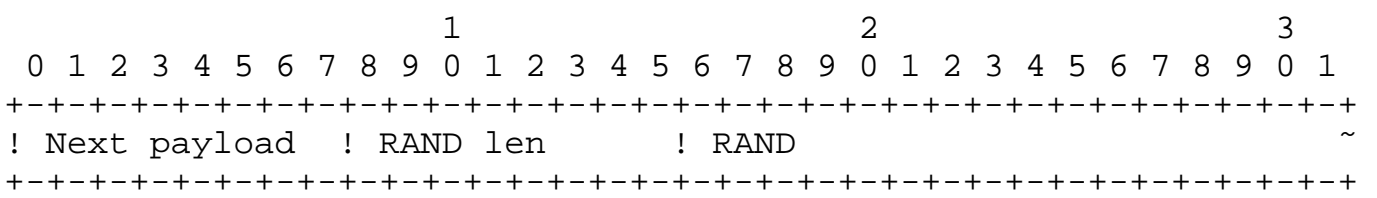

* Next payload ( 8 bits): identifies the payload that is added after this payload. See section 6.1 for values.

* RAND len ( 8 bits): length of the RAND (in bytes). It SHOULD be at least 16 .

* RAND (variable length): a (pseudo-) randomly chosen bit-string. 


\subsection{Error payload (ERR)}

The Error payload is used to specify the error(s) that may have occurred.

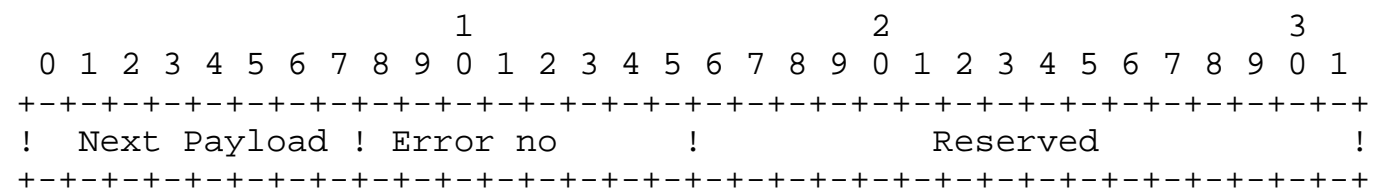

* Next payload ( 8 bits): identifies the payload that is added after this payload. See Section 6.1 for values.

* Error no (8 bits): indicates the type of error that was encountered.

\begin{tabular}{l|r|l} 
Error no & Value & Comment \\
Auth failure & 0 & Authentication failure \\
Invalid TS & 1 & Invalid timestamp \\
Invalid PRF & 2 & PRF function not supported \\
Invalid MAC & 3 & MAC algorithm not supported \\
Invalid EA & 4 & Encryption algorithm not supported \\
Invalid HA & 5 & Hash function not supported \\
Invalid DH & 6 & DH group not supported \\
Invalid ID & 7 & ID not supported \\
Invalid Cert & 8 & Certificate not supported \\
Invalid SP & 9 & SP type not supported \\
Invalid SPpar & 10 & SP parameters not supported \\
Invalid DT & 11 & not supported Data type \\
Unspecified error & 12 & an unspecified error occurred \\
Table 6.12 &
\end{tabular}

\subsection{Key data sub-payload}

The Key data payload contains key material, e.g., TGKs. The Key data payloads are never included in clear, but as an encrypted part of the Key data transport payload.

Note that a Key data transport payload can contain multiple Key data sub-payloads. 


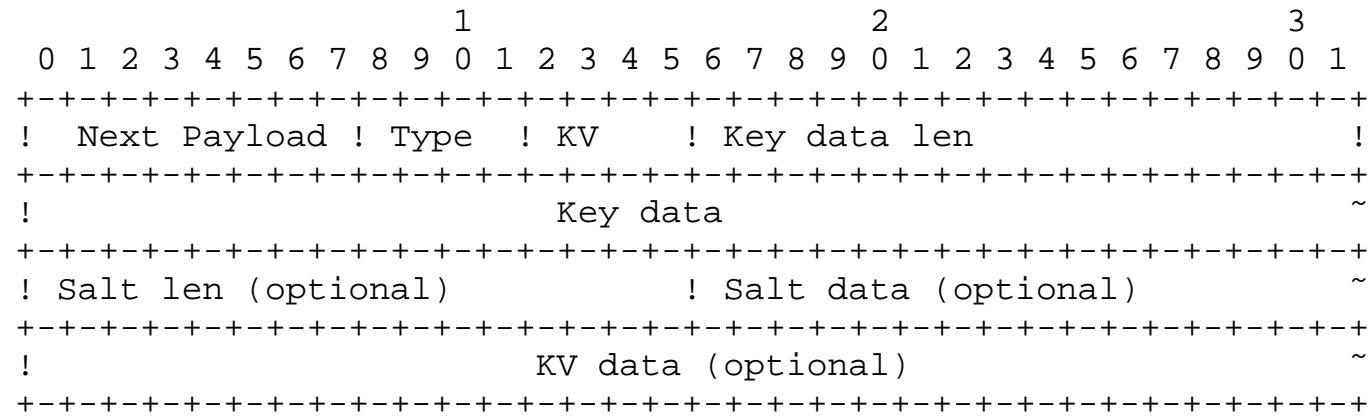

* Next payload ( 8 bits): identifies the payload that is added after this payload. See section 6.1 for values.

* Type (4 bits): indicates the type of key included in the payload.

\begin{tabular}{l|c} 
Type & Value \\
\hline-----1 & 0 \\
TGK & 1 \\
TGK+SALT & 2 \\
TEK & 3 \\
TEK+SALT & 3
\end{tabular}

Table 6.13.a

Note that the possibility of including a TEK (instead of using the TGK) is provided. When sent directly, the TEK can generally not be shared between more than one Crypto Session (unless the Security protocol allows for this, e.g., [SRTP]). The recommended use of sending a TEK, instead of a TGK, is when pre-encrypted material exists and therefore, the TEK must be known in advance.

* KV (4 bits): indicates the type of key validity period specified. This may be done by using an SPI (or MKI in the case of [SRTP]) or by providing an interval in which the key is valid (e.g., in the latter case, for SRTP this will be the index range where the key is valid). 


\begin{tabular}{|c|c|c|}
\hline KV & Value & Comments \\
\hline Null & 0 & $\begin{array}{l}\text { No specific usage rule (e.g., a TEK } \\
\text { that has no specific lifetime) }\end{array}$ \\
\hline SPI & 1 & The key is associated with the SPI/MKI \\
\hline Interval & 2 & $\begin{array}{l}\text { The key has a start and expiration time } \\
(e . g \cdot, \text { an SRTP TEK) }\end{array}$ \\
\hline
\end{tabular}

Table 6.13.b

Note that when NULL is specified, any SPI or Interval is valid. For an Interval, this means that the key is valid from the first observed sequence number until the key is replaced (or the security protocol is shutdown).

* Key data len (16 bits): the length of the Key data field (in bytes). Note that the sum of the overall length of all the Key data payloads contained in a single key data transport payload (KEMAC) MUST be such that the KEMAC payload does not exceed a length of $2^{\wedge} 16$ bytes (total length of KEMAC, see Section 6.2).

* Key data (variable length): The TGK or TEK data.

* Salt len (16 bits): The salt key length in bytes. Note that this field is only included if the salt is specified in the Type-field.

* Salt data (variable length): The salt key data. Note that this field is only included if the salt is specified in the Type-field. (For SRTP, this is the so-called master salt.)

* KV data (variable length): This includes either the SPI or an interval (see Section 6.14). If KV is NULL, this field is not included.

\subsection{Key validity data}

The Key validity data is not a standalone payload, but part of either the Key data payload (see Section 6.13) or the DH payload (see Section 6.4). The Key validity data gives a guideline of when the key should be used. There are two KV types defined (see Section 6.13), SPI/MKI (SPI) or a lifetime range (interval). 
SP I / MKI

$\begin{array}{llllllllllllllllllllllllllllllll}0 & 1 & 2 & 3 & 4 & 5 & 6 & 7 & 8 & 9 & 0 & 1 & 2 & 3 & 4 & 5 & 6 & 7 & 8 & 9 & 0 & 1 & 2 & 3 & 4 & 5 & 6 & 7 & 8 & 9 & 0 & 1\end{array}$ +-+-+-+-+-+-+-+-+-+-+-+-+-+-+-+-+-+-+-+-+-+-+-+-+-+-+-+-+-+-+-+-+ ! SPI Length ! SPI

* SPI Length (8 bits): the length of the SPI (or MKI) in bytes.

* SPI (variable length): the SPI (or MKI) value.

Interval

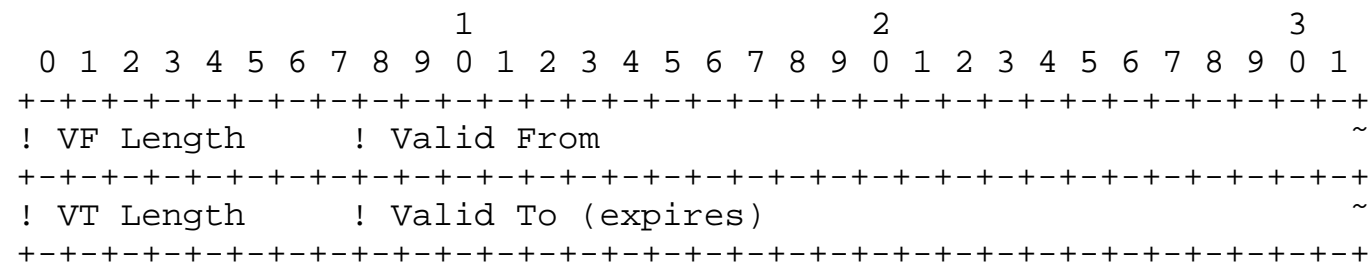

* VF Length (8 bits): length of the Valid From field in bytes.

* Valid From (variable length): sequence number, index, timestamp, or other start value that the security protocol uses to identify the start position of the key usage.

* VT Length (8 bits): length of the Valid To field in bytes.

* Valid To (variable length): sequence number, index, timestamp, or other expiration value that the security protocol can use to identify the expiration of the key usage.

Note that for SRTP usage, the key validity period for a TGK/TEK should be specified with either an interval, where the VF/VT Length is equal to 6 bytes (i.e., the size of the index), or with an MKI. It is RECOMMENDED that if more than one SRTP stream is sharing the same keys and key update/re-keying is desired, this is handled using MKI rather than the From-To method. 


\subsection{General Extension Payload}

The General extensions payload is included to allow possible extensions to MIKEY without the need for defining a completely new payload each time. This payload can be used in any MIKEY message and is part of the authenticated/signed data part.

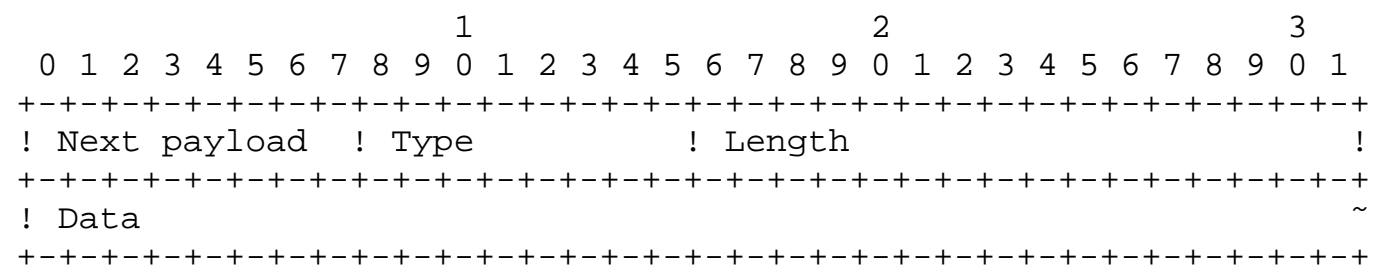

* Next payload (8 bits): identifies the payload that is added after this payload.

* Type (8 bits): identifies the type of general payload.

\begin{tabular}{l|l|l|l} 
Type & Value & Comments \\
- & \\
Vendor ID & 0 & Vendor specific byte string \\
SDP IDs & 1 & List of SDP key mgmt IDs (allocated for use in
\end{tabular}
[KMASDP ] )

Table 6.15

* Length (16 bits): the length in bytes of the Data field.

* Data (variable length): the general payload data.

7. Transport protocols

MIKEY MAY be integrated within session establishment protocols. Currently, integration of MIKEY within SIP/SDP and RTSP is defined in [KMASDP]. MIKEY MAY use other transports, in which case how MIKEY is transported over such a transport protocol has to be defined.

8. Groups

What has been discussed up to now is not limited to single peer-topeer communication (except for the DH method), but can be used to distribute group keys for small-size interactive groups and simple one-to-many scenarios. Section 2.1. describes the scenarios in the focus of MIKEY. This section describes how MIKEY is used in a group scenario (though, see also section 4.3 for issues related to authorization). 


\subsection{Simple one-to-many}

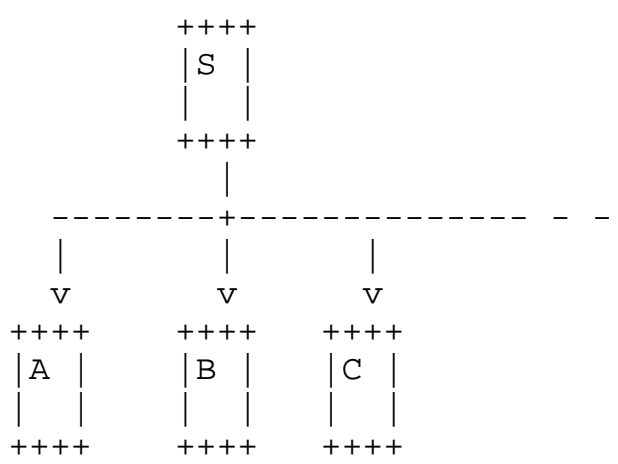

Figure 8.1. Simple one-to-many scenario.

In the simple one-to-many scenario, a server is streaming to a small group of clients. RTSP or SIP is used for the registration and the key management set up. The streaming server acts as the Initiator of MIKEY. In this scenario, the pre-shared key or public key transport mechanism will be appropriate in transporting the same TGK to all the clients (which will result in common TEKs for the group).

Note, if the same TGK/TEK(s) should be used by all the group members, the streaming server MUST specify the same CSB_ID and CS_ID(s) for the session to all the group members.

As the communication may be performed using multicast, the members need a common security policy if they want to be part of the group. This limits the possibility of negotiation.

Furthermore, the Initiator should carefully consider whether to request the verification message in reply from each receiver, as this may result in a certain load for the Initiator itself as the group size increases.

\subsection{Small-size interactive group}

As described in the overview section, for small-size interactive groups, one may expect that each client will be in charge for setting up the security for its outgoing streams. In these scenarios, the pre-shared key or the public-key transport method is used. 


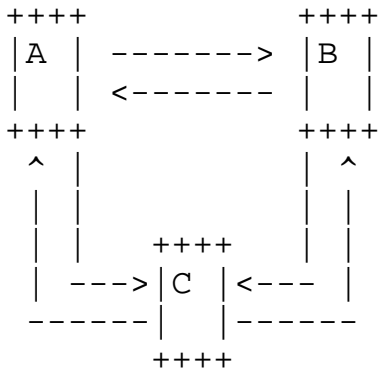

Figure 8.2. Small-size group without a centralized controller.

One scenario may then be that the client sets up a three-part call, using SIP. Due to the small size of the group, unicast SRTP is used between the clients. Each client sets up the security for its outgoing stream(s) to the others.

As for the simple one-to-many case, the streaming client specifies the same CSB_ID and CS_ID(s) for its outgoing sessions if the same TGK/TEK $(s)$ is used for all the group members.

9. Security Considerations

\subsection{General}

Key management protocols based on timestamps/counters and oneroundtrip key transport have previously been standardized, for example ISO [ISO1, ISO2]. The general security of these types of protocols can be found in various articles and literature, c.f. [HAC, $\mathrm{AKE}, \mathrm{LOA}]$.

No chain is stronger than its weakest link. If a given level of protection is wanted, then the cryptographic functions protecting the keys during transport/exchange MUST offer a security corresponding to at least that level.

For instance, if a security against attacks with a complexity $2 \wedge 96$ is wanted, then one should choose a secure symmetric cipher supporting at least 96 bit keys (128 bits may be a practical choice) for the actual media protection, and a key transport mechanism that provides equivalent protection, e.g., MIKEY's pre-shared key transport with 128 bit TGK, or RSA with 1024 bit keys (which according to [LV] corresponds to the desired 96 bit level, with some margin).

In summary, key size for the key-exchange mechanism MUST be weighed against the size of the exchanged TGK so that it at least offers the required level. For efficiency reasons, one SHOULD also avoid a 
security overkill, e.g., by not using a public key transport with public keys giving a security level that is orders of magnitude higher than length of the transported TGK. We refer to [LV] for concrete key size recommendations.

Moreover, if the TGKs are not random (or pseudo-random), a brute force search may be facilitated, again lowering the effective key size. Therefore, care MUST be taken when designing the (pseudo-) random generators for TGK generation, see [FIPS][RAND].

For the selection of the hash function, SHA-1 with 160-bit output is the default one. In general, hash sizes should be twice the "security level", indicating that SHA-1-256, [SHA256], should be used for the default 128-bit level. However, due to the real-time aspects in the scenarios we are treating, hash sizes slightly below 256 are acceptable, as the normal "existential" collision probabilities would be of secondary importance.

In a Crypto Session Bundle, the Crypto Sessions can share the same TGK as discussed earlier. From a security point of view, to satisfy the criterion in case the TGK is shared, the encryption of the individual Crypto Sessions are performed "independently". In MIKEY, this is accomplished by having unique Crypto Session identifiers (see also section 4.1) and a TEK derivation method that provides cryptographically independent TEKs to distinct Crypto Sessions (within the Crypto Session Bundle), regardless of the security protocol used.

Specifically, the key derivations, as specified in section 4.1, are implemented by a pseudo-random function. The one used here is a simplified version of that used in TLS [TLS]. Here, only one single hash function is used, whereas TLS uses two different functions. This choice is motivated by the high confidence in the SHA-1 hash function, and by efficiency and simplicity of design (complexity does not imply security). Indeed, as shown in [DBJ], if one of the two hashes is severely broken, the TLS PRF is actually less secure than as if a single hash had been used on the whole key, as is done in MIKEY.

In the pre-shared key and public-key schemes, the TGK is generated by a single party (Initiator). This makes MIKEY somewhat more sensitive if the Initiator uses a bad random number generator. It should also be noted that neither the pre-shared nor the public-key scheme provides perfect forward secrecy. If mutual contribution or perfect forward secrecy is desired, the Diffie-Hellman method is to be used. Authentication (e.g., signatures) in the Diffie-Hellman method is required to prevent man-in-the-middle attacks. 
Forward/backward security: if the TGK is exposed, all generated TEKs are compromised. However, under the assumption that the derivation function is a pseudo-random function, disclosure of an individual TEK does not compromise other (previous or later) TEKs derived from the same TGK. The Diffie-Hellman mode can be considered by cautious users, as it is the only one that supports so called perfect forward secrecy (PFS). This is in contrast to a compromise of the pre-shared key (or the secret key of the public key mode), where future sessions and recorded sessions from the past are then also compromised.

The use of random nonces (RANDs) in the key derivation is of utmost importance to counter off-line pre-computation attacks. Note however that update messages re-use the old RAND. This means that the total effective key entropy (relative to pre-computation attacks) for $\mathrm{k}$ consecutive key updates, assuming the TGKs and RAND are each $\mathrm{n}$ bits long, is about $\mathrm{L}=\mathrm{n} *(\mathrm{k}+1) / 2$ bits, compared to the theoretical maximum of $\mathrm{n}^{\star} \mathrm{k}$ bits. In other words, a $2^{\wedge} \mathrm{L}$ work effort MAY enable an attacker to get all $\mathrm{k}$ n-bit keys, which is better than brute force (except when $\mathrm{k}=1$ ). While this might seem like a defect, first note that for a proper choice of $n$, the $2^{\wedge} \mathrm{L}$ complexity of the attack is way out of reach. Moreover, the fact that more than one key can be compromised in a single attack is inherent to the key exchange problem. Consider for instance a user who, using a fixed 1024-bit RSA key, exchanges keys and communicates during a one or two year lifetime of the public key. Breaking this single RSA key will enable access to all exchanged keys and consequently the entire communication of that user over the whole period.

All the pre-defined transforms in MIKEY use state-of-the-art algorithms that have undergone large amounts of public evaluation. One of the reasons for using the AES-CM from SRTP [SRTP], is to have the possibility of limiting the overall number of different encryption modes and algorithms, while offering a high level of security at the same time.

\subsection{Key lifetime}

Even if the lifetime of a TGK (or TEK) is not specified, it MUST be taken into account that the encryption transform in the underlying security protocol can in some way degenerate after a certain amount of encrypted data. It is not possible to here state universally applicable, general key lifetime bounds; each security protocol should define such maximum amount and trigger a re-keying procedure before the "exhaustion" of the key. For example, according to SRTP [SRTP] the TEK, together with the corresponding TGK, MUST be changed at least every $2^{\wedge} 48$ SRTP packet. 
Still, the following can be said as a rule of thumb. If the security protocol uses an "ideal" b-bit block cipher (in CBC mode, counter mode, or a feedback mode, e.g., OFB, with full b-bit feedback), degenerate behavior in the crypto stream, possibly useful for an attacker, is (with constant probability) expected to occur after a total of roughly $2^{\wedge}(\mathrm{b} / 2)$ encrypted b-bit blocks (using random IVs). For security margin, re-keying MUST be triggered well in advance compared to the above bound. See [BDJR] for more details.

For use of a dedicated stream cipher, we refer to the analysis and documentation of said cipher in each specific case.

\subsection{Timestamps}

The use of timestamps, instead of challenge-responses, requires the systems to have synchronized clocks. Of course, if two clients are not synchronized, they will have difficulties in setting up the security. The current timestamp based solution has been selected to allow a maximum of one roundtrip (i.e., two messages), but still provide a reasonable replay protection. A (secure) challengeresponse based version would require at least three messages. For a detailed description of the timestamp and replay handling in MIKEY, see section 5.4 .

Practical experiences of Kerberos and other timestamp-based systems indicate that it is not always necessary to synchronize the terminals over the network. Manual configuration could be a feasible alternative in many cases (especially in scenarios where the degree of looseness is high). However, the choice must be made carefully with respect to the usage scenario.

\subsection{Identity Protection}

User privacy is a complex matter that to some extent can be enforced by cryptographic mechanisms, but also requires policy enforcement and various other functionalities. One particular facet of privacy is user identity protection. However, identity protection was not a main design goal for MIKEY. Such a feature will add more complexity to the protocol and was therefore not chosen to be included. As MIKEY is anyway proposed to be transported over, e.g., SIP, the identity may be exposed by this. However, if the transporting protocol is secured and also provides identity protection, MIKEY might inherit the same feature. How this should be done is for future study. 


\subsection{Denial of Service}

This protocol is resistant to Denial of Service attacks in the sense that a Responder does not construct any state (at the key management protocol level) before it has authenticated the Initiator. However, this protocol, like many others, is open to attacks that use spoofed IP addresses to create a large number of fake requests. This may for example, be solved by letting the protocol transporting MIKEY do an IP address validity test. The SIP protocol can provide this using the anonymous authentication challenge mechanism (specified in Section 22.1 of [SIP]).

It is highly RECOMMENDED to include IDr in the Initiator's message. If not included, its absence can be used for Dos purposes (the largest Dos-impact being on the public key and DH methods), where a message intended for other entities is sent to the target. In fact, the target may verify the signature correctly due to the fact that the Initiator's ID is correct and the message is actually signed by the claimed Initiator (e.g., by re-directing traffic from another session).

However, in the public key method, the envelop key and the MAC will ensure that the message is not accepted (still, compared to a normal faked message, where the signature verification would detect the problem, one extra public key decryption is needed to detect the problem in this case).

In the DH method, a message would be accepted (without detecting the error) and a response (and state) would be created for the malicious request.

As also discussed in Section 5.4, the tradeoff between time synchronization and the size of the replay cache may be affected in case of for example, a flooding Dos attack. However, if the recommendations of using a dynamic size of the replay cache are followed, it is believed that the client will in most cases be able to handle the replay cache. Of course, as the replay cache decreases in size, the required time synchronization is more restricted. However, a bigger problem during such an attack would probably be to process the messages (e.g., verify signatures/MACs) due to the computational workload this implies.

\subsection{Session Establishment}

It should be noted that if the session establishment protocol is insecure, there may be attacks on this that will have indirect security implications on the secured media streams. This however only applies to groups (and is not specific to MIKEY). The threat is 
that one group member may re-direct a stream from one group member to another. This will have the same implication as when a member tries to impersonate another member, e.g., by changing its IP address. If this is seen as a problem, it is RECOMMENDED that a Data Origin Authentication (DOA) scheme (e.g., digital signatures) be applied to the security protocol.

Re-direction of streams can of course be done even if it is not a group. However, the effect will not be the same as compared to a group where impersonation can be done if DOA is not used. Instead, re-direction will only deny the receiver the possibility of receiving (or just delay) the data.

10. IANA Considerations

This document defines several new name spaces associated with the MIKEY payloads. This section summarizes the name spaces for which IANA is requested to manage the allocation of values. IANA is requested to record the pre-defined values defined in the given sections for each name space. IANA is also requested to manage the definition of additional values in the future. Unless explicitly stated otherwise, values in the range 0-240 for each name space SHOULD be approved by the process of IETF consensus and values in the range 241-255 are reserved for Private Use, according to [RFC2434].

The name spaces for the following fields in the Common header payload (from Section 6.1) are requested to be managed by IANA (in bracket is the reference to the table with the initially registered values):

* version

* data type (Table 6.1.a)

* Next payload (Table 6.1.b)

* PRF func (Table 6.1.c). This name space is between 0-127, where values between 0-111 should be approved by the process of IETF consensus and values between 112-127 are reserved for Private Use.

* CS ID map type (Table 6.1.d)

The name spaces for the following fields in the Key data transport payload (from section 6.2) are requested to be managed by IANA:

* Encr alg (Table 6.2.a)

* MAC alg (Table 6.2.b) 
The name spaces for the following fields in the Envelope data payload (from section 6.3) are requested to be managed by IANA:

* C (Table 6.3)

The name spaces for the following fields in the DH data payload (from Section 6.4) are requested to be managed by IANA:

* DH-Group (Table 6.4)

The name spaces for the following fields in the Signature payload (from Section 6.5) are requested to be managed by IANA:

* S type (Table 6.5)

The name spaces for the following fields in the Timestamp payload (from Section 6.6) are requested to be managed by IANA:

* TS type (Table 6.6)

The name spaces for the following fields in the ID payload and the Certificate payload (from Section 6.7) are requested to be managed by IANA :

* ID type (Table 6.7.a)

* Cert type (Table 6.7.b)

The name spaces for the following fields in the Cert hash payload (from Section 6.8) are requested to be managed by IANA:

* Hash func (Table 6.8)

The name spaces for the following fields in the Security policy payload (from section 6.10) are requested to be managed by IANA:

* Prot type (Table 6.10)

For each security protocol that uses MIKEY, a set of unique parameters MAY be registered.

From Section 6.10.1.

* SRTP Type (Table 6.10.1.a)

* SRTP encr alg (Table 6.10.1.b)

* SRTP auth alg (Table 6.10.1.c) 
* SRTP PRF (Table 6.10.1.d)

* FEC order (Table 6.10.1.e)

The name spaces for the following fields in the Error payload (from Section 6.12) are requested to be managed by IANA:

* Error no (Table 6.12)

The name spaces for the following fields in the Key data payload (from Section 6.13) are requested to be managed by IANA:

* Type (Table 6.13.a). This name space is between 0-16, which should be approved by the process of IETF consensus.

* KV (Table 6.13.b). This name space is between 0-16, which should be approved by the process of IETF consensus.

The name spaces for the following fields in the General Extensions payload (from section 6.15) are requested to be managed by IANA:

* Type (Table 6.15).

10.1. MIME Registration

This section gives instructions to IANA to register the application/mikey MIME media type. This registration is as follows:

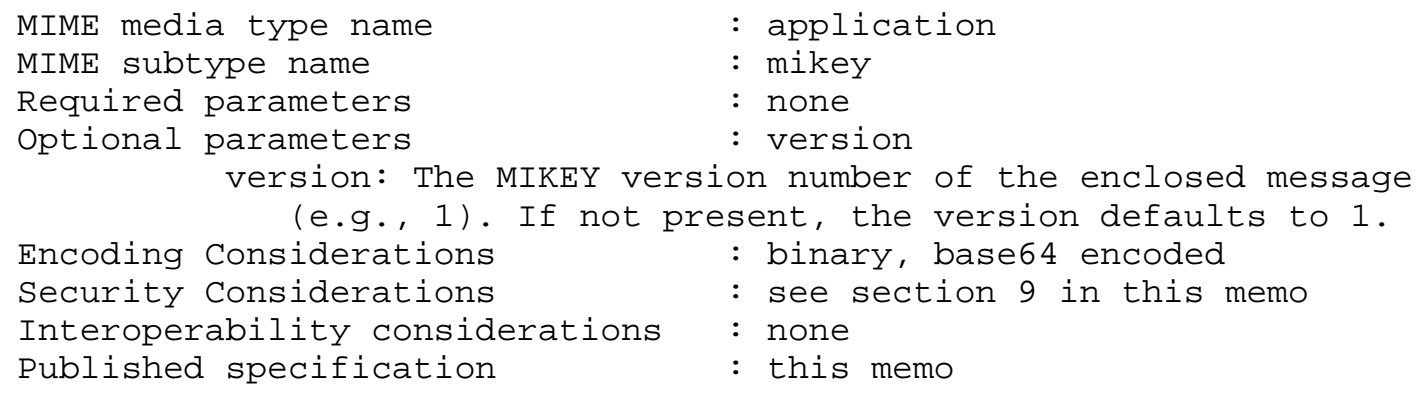

11. Acknowledgments

The authors would like to thank Mark Baugher, Ran Canetti, Martin Euchner, Steffen Fries, Peter Barany, Russ Housley, Pasi Ahonen (with his group), Rolf Blom, Magnus Westerlund, Johan Bilien, Jon-Olov Vatn, Erik Eliasson, and Gerhard Strangar for their valuable feedback. 
12. References

12.1. Normative References

[HMAC] Krawczyk, H., Bellare, M., and R. Canetti, "HMAC: KeyedHashing for Message Authentication", RFC 2104, February 1997 .

[NAI] Aboba, B. and M. Beadles, "The Network Access Identifier", RFC 2486, January 1999.

[OAKLEY] Orman, H., "The OAKLEY Key Determination Protocol", RFC 2412, November 1998.

[PSS] PKCS \#1 v2.1 - RSA Cryptography Standard, RSA Laboratories, June 14, 2002, wWw.rsalabs.com

[RFC2119] Bradner, S., "Key words for use in RFCs to Indicate Requirement Levels", BCP 14, RFC 2119, March 1997.

[RFC2434] Narten, $\mathrm{T}$. and H. Alvestrand, "Guidelines for Writing an IANA Considerations section in RFCs", BCP 26, RFC 2434, October 1998 .

[SHA-1] NIST, FIPS PUB 180-1: Secure Hash Standard, April 1995.

[SRTP] Baugher, M., MCGrew, D., Naslund, M., Carrara, E., and K. Norrman, "The Secure Real Time Transport Protocol", RFC 3711, March 2004.

[URI] Berners-Lee, T., Fielding, R., and L. Masinter, "Uniform Resource Identifiers (URI) : Generic Syntax", RFC 2396 , August 1998 .

[X.509] Housley, R., Polk, W., Ford, W., and D. Solo, "Internet X.509 Public Key Infrastructure Certificate and Certificate Revocation List (CRL) Profile", RFC 3280, April 2002.

[AESKW] Schaad, J. and R. Housley, "Advanced Encryption Standard (AES) Key Wrap Algorithm", RFC 3394, september 2002. 


\subsection{Informative References}

[AKE] Canetti, R. and H. Krawczyk, "Analysis of Key-Exchange Protocols and their use for Building Secure Channels", Eurocrypt 2001, LNCS 2054, pp. 453-474, 2001.

[BDJR] Bellare, M., Desai, A., Jokipii, E., and P. Rogaway, "A Concrete Analysis of Symmetric Encryption: Analysis of the DES Modes of Operation", in Proceedings of the 38th Symposium on Foundations of Computer Science, IEEE, 1997, pp. 394-403.

[BMGL] Hastad, J. and M. Naslund: "Practical Construction and Analysis of Pseduo-randomness Primitives", Proceedings of Asiacrypt 2001, LNCS. vol 2248, pp. 442-459, 2001.

[DBJ] Johnson, D.B., "Theoretical Security Concerns with TLS use of MD5", Contribution to ANSI X9F1 WG, 2001.

[FIPS] "Security Requirements for Cryptographic Modules", Federal Information Processing Standard Publications (FIPS PUBS) 140-2, December 2002 .

[GKMARCH] Baugher, M., Canetti, R., Dondeti, L., and F. Lindholm, "Group Key Management Architecture", Work in Progress.

[GDOI] Baugher, M., Weis, B., Hardjono, T., and H. Harney, "The Group Domain of Interpretation", RFC 3547, July 2003.

[GSAKMP] Harney, H., Colegrove, A., Harder, E., Meth, U., and R. Fleischer, "Group Secure Association Key Management Protocol", Work in Progress.

[HAC] Menezes, A., van Oorschot, P., and S. Vanstone, "Handbook of Applied Cryptography", CRC press, 1996.

[IKE] Harkins, D. and D. Carrel, "The Internet Key Exchange (IKE)", RFC 2409, November 1998.

[ISO1] ISO/IEC 9798-3: 1997, Information technology - Security techniques - Entity authentication - Part 3: Mechanisms using digital signature techniques.

[ISO2] ISO/IEC 11770-3: 1997, Information technology - Security techniques - Key management - Part 3: Mechanisms using digital signature techniques. 
[ISO3] ISO/IEC 18014 Information technology - Security techniques - Time-stamping services, Part 1-3.

[KMASDP] Arkko, J., Carrara, E., Lindholm, F., Naslund, M., and K. Norrman, "Key Management Extensions for SDP and RTSP", Work in Progress.

[LOA] Burrows, Abadi, and Needham, "A logic of authentication", ACM Transactions on Computer Systems 8 No.1 (Feb. 1990), $18-36$.

[LV] Lenstra, A. K. and E. R. Verheul, "Suggesting Key Sizes for Cryptosystems", http://www.cryptosavvy.com/suggestions.htm

[NTP] Mills, D., "Network Time Protocol (Version 3) Specification, Implementation and Analysis", RFC 1305, March 1992 .

[OCSP] Myers, M., Ankney, R., Malpani, A., Galperin, S., and C. Adams, "X.509 Internet Public Key Infrastructure Online Certificate Status Protocol - OCSP", RFC 2560, June 1999.

[RAND] Eastlake, 3rd, D., Crocker, S., and J. Schiller, "Randomness Requirements for Security", RFC 1750, December 1994 .

[RTSP] Schulzrinne, H., Rao, A., and R. Lanphier, "Real Time Streaming Protocol (RTSP)", RFC 2326, April 1998.

[SDP] Handley, M. and V. Jacobson, "SDP: Session Description Protocol", RFC 2327, April 1998.

[SHA256] NIST, "Description of SHA-256, SHA-384, and SHA-512", http://csrc.nist.gov/encryption/shs/sha256-384-512.pdf

[SIP] Rosenberg, J., Schulzrinne, H., Camarillo, G., Johnston, A., Peterson, J., Sparks, R., Handley, M., and E. Schooler, "SIP: Session Initiation Protocol", RFC 3261, June 2002.

[TLS] Dierks, T. and C. Allen, "The TLS Protocol - Version 1.0", RFC 2246, January 1999. 
Appendix A. MIKEY - SRTP Relation

The terminology in MIKEY differs from the one used in SRTP as MIKEY needs to be more general, nor is tight to SRTP only. Therefore, it might be hard to see the relations between keys and parameters generated in MIKEY and those used by SRTP. This section provides some hints on their relation.

\begin{tabular}{l|l} 
MIKEY & SRTP \\
\begin{tabular}{l|l} 
Crypto Session \\
Data SA
\end{tabular} & $\begin{array}{l}\text { SRTP stream (typically with related SRTCP stream) } \\
\text { TEK }\end{array}$
\end{tabular}

The Data SA is built up by a TEK and the security policy exchanged. SRTP may use an MKI to index the TEK or TGK (the TEK is then derived from the TGK that is associated with the corresponding MKI), see below.

\section{A.1. MIKEY-SRTP Interactions}

In the following, we give a brief outline of the interface between SRTP and MIKEY and the processing that takes place. We describe the SRTP receiver side only, the sender side will require analogous interfacing.

1. When an SRTP packet arrives at the receiver and is processed, the triple <SSRC, destination address, destination port> is extracted from the packet and used to retrieve the correct SRTP crypto context, hence the Data SA. (The actual retrieval can, for example, be done by an explicit request from the SRTP implementation to MIKEY, or, by the SRTP implementation accessing a "database", maintained by MIKEY. The application will typically decide which implementation is preferred.)

2. If an MKI is present in the SRTP packet, it is used to point to the correct key within the SA. Alternatively, if SRTP's <From, To> feature is used, the ROC||SEQ of the packet is used to determine the correct key.

3. Depending on whether the key sent in MIKEY (as obtained in step 2) was a TEK or a TGK, there are now two cases.

- If the key obtained in step 2 is the TEK itself, it is used directly by SRTP as a master key. 
- If the key instead is a TGK, the mapping with the CS_ID (internal to MIKEY, Section 6.1.1) allows MIKEY to compute the correct TEK from the TGK as described in section 4.1 before SRTP uses it.

If multiple TGKs (or TEKs) are sent, it is RECOMMENDED that each TGK (or TEK) be associated with a distinct MKI. It is RECOMMENDED that the use of <From, To> in this scenario be limited to very simple cases, e.g., one stream only.

Besides the actual master key, other information in the Data SA (e.g., transform identifiers) will of course also be communicated from MIKEY to SRTP. 


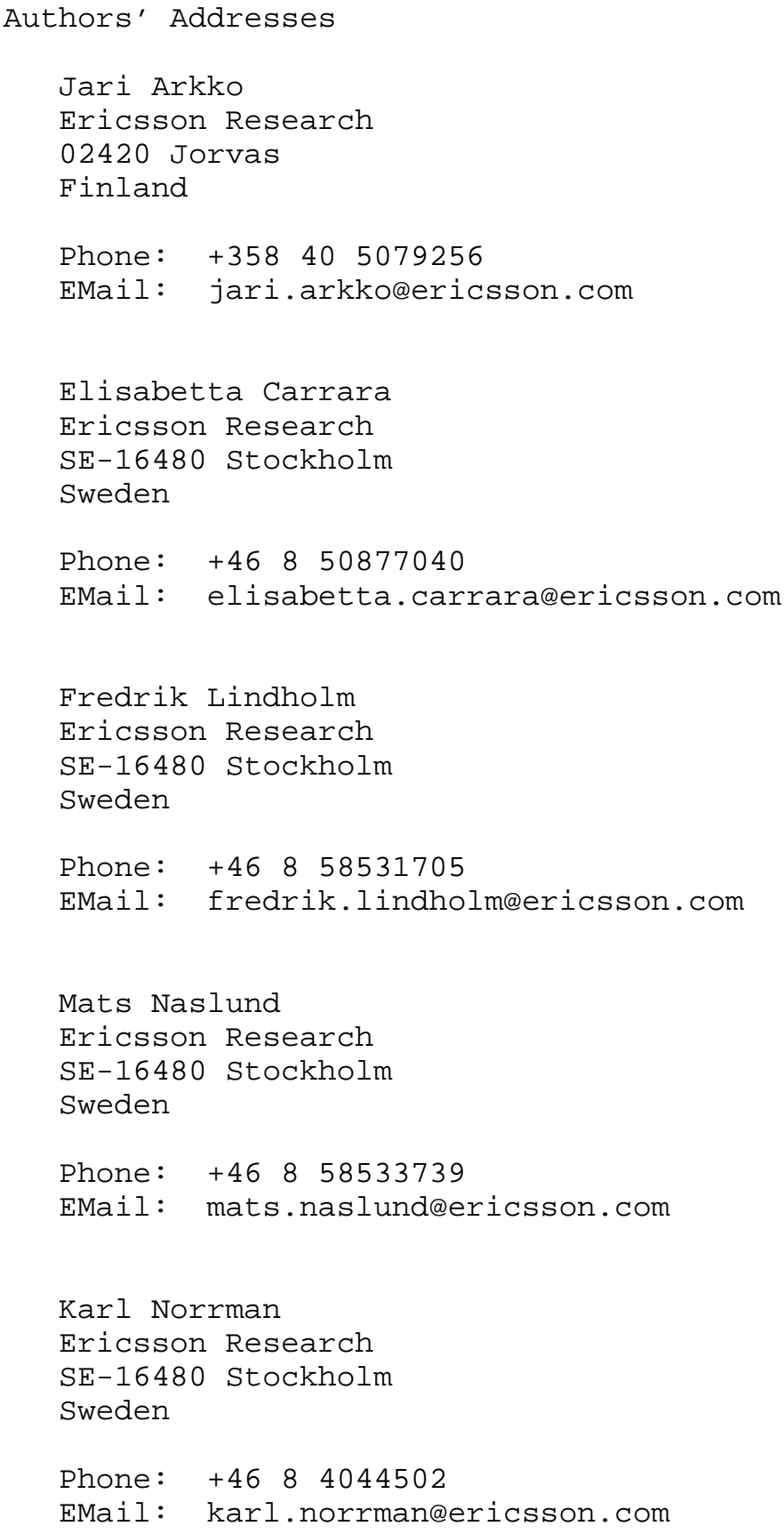


Full Copyright statement

Copyright (C) The Internet Society (2004). This document is subject to the rights, licenses and restrictions contained in BCP 78, and except as set forth therein, the authors retain all their rights.

This document and the information contained herein are provided on an "AS IS" basis and THE CONTRIBUTOR, THE ORGANIZATION HE/SHE REPRESENTS OR IS SPONSORED BY (IF ANY), THE INTERNET SOCIETY AND THE INTERNET ENGINEERING TASK FORCE DISCLAIM ALL WARRANTIES, EXPRESS OR IMPLIED, INCLUDING BUT NOT LIMITED TO ANY WARRANTY THAT THE USE OF THE INFORMATION HEREIN WILL NOT INERINGE ANY RIGHTS OR ANY IMPLIED WARRANTIES OF MERCHANTABILITY OR FITNESS FOR A PARTICULAR PURPOSE.

Intellectual Property

The IETF takes no position regarding the validity or scope of any Intellectual Property Rights or other rights that might be claimed to pertain to the implementation or use of the technology described in this document or the extent to which any license under such rights might or might not be available; nor does it represent that it has made any independent effort to identify any such rights. Information on the procedures with respect to rights in RFC documents can be found in BCP 78 and BCP 79 .

Copies of IPR disclosures made to the IETF Secretariat and any assurances of licenses to be made available, or the result of an attempt made to obtain a general license or permission for the use of such proprietary rights by implementers or users of this specification can be obtained from the IETF on-line IPR repository at http://www.ietf.org/ipr.

The IETF invites any interested party to bring to its attention any copyrights, patents or patent applications, or other proprietary rights that may cover technology that may be required to implement this standard. Please address the information to the IETF at ietfipreietf.org.

Acknowledgement

Funding for the RFC Editor function is currently provided by the Internet society. 